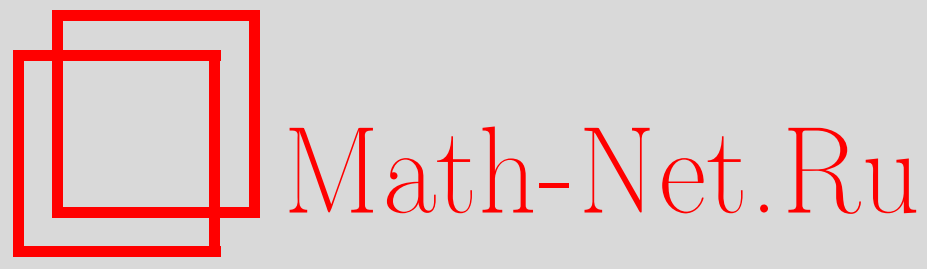

Ph. Barbe, M. Broniatowski, On sharp large deviations for sums of random vectors and multidimensional Laplace approximation, Теория вероятн. и ее примен., 2004, том 49, выпуск 4, 743-774

DOI: https://doi.org/10.4213/tvp192

Использование Общероссийского математического портала Math-Net.Ru подразумевает, что вы прочитали и согласны с пользовательским соглашением

http: //www . mathnet.ru/rus/agreement

Параметры загрузки:

IP : 52.205.19.152

26 апреля 2023 г., 14:14:24

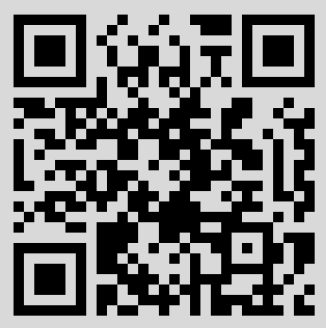




\section{ON SHARP LARGE DEVIATIONS FOR SUMS OF RANDOM VECTORS AND MULTIDIMENSIONAL LAPLACE APPROXIMATION}

Пусть $X, X_{i}, i \geqslant 1$, - последовательность независимых одинаково распределенных векторов в $\mathbf{R}^{d}$. Рассмотрим частичные суммы $S_{n}:=X_{1}+\cdots+X_{n}$. При некоторых условиях регулярности на распределение $X$ мы получаем асимптотическую формулу для $P\left\{S_{n} \in n A\right\}$, где $A-$ произвольное борелевское множество. Приводится несколько следствий, одно из которых утверждает, что, при тех же условиях регулярности, для любого борелевского множества $A$ предел $\lim _{n \rightarrow \infty} n^{-1} \ln P\left\{S_{n} \in n A\right\}=-I(A)$, где $I-$ функционал больших уклонений. Мы также доказываем результат о многомерной аппроксимации типа Лапласа, который позволяет явно вычислить вероятности точных больших уклонений, когда $A$ имеет гладкую границу.

Ключевые слова и фразы: большие уклонения, экспоненциальное семейство, дифференциальная геометрия поверхностей, асимптотический анализ, метод Лапласа, преобразование Фурье.

1. Introduction and notation. Consider a sequence $X, X_{i}, i \geqslant 1$, of independent and identically distributed random variables in $\mathbf{R}^{d}, d \geqslant 1$. Let $S_{n}:=\sum_{1 \leqslant i \leqslant n} X_{i}$, so that $S_{n} / n$ is just the mean of $X_{1}, \ldots, X_{n}$.

If $E|X|<\infty$, where $|\cdot|$ denotes the usual norm in $\mathbf{R}^{d}$, the strong law of large numbers asserts that $S_{n} / n$ converges almost surely to the expectation $E X$. One of the purposes of large deviation probabilities is somewhat to quantify this convergence. It consists in estimating probabilities $P\left\{S_{n} \in n A\right\}$, where $A$ is a Borel set which typically does not contain $E X$. The Russian literature introduced a useful distinction between what is called rough large deviations, where one is interested in estimating $\ln P\left\{S_{n} \in n A\right\}$, and the so-called precise (or sharp) large deviations, where one is interested in finding asymptotic equivalent for $P\left\{S_{n} \in n A\right\}$.

A lot is known about rough large deviations, and it is common (since somewhat necessary — see [37]) to assume that the moment generating function

$$
\phi(t):=E \exp \langle t, X\rangle \quad \text { is finite for small } t
$$

* CNRS, 90 rue de Vaugirard, 75006 Paris, France; e-mail: ph_barbe@hotmail.com

** Université de Reims and CNRS e-mail: mbr@ccr.jussieu.fr 
where $\langle\cdot, \cdot\rangle$ is the usual inner product in $\mathbf{R}^{d}$. Under (1.1), one defines for every $x \in \mathbf{R}^{d}$ the convex conjugate of $\ln \phi$,

$$
I(x):=\sup \left\{\langle t, x\rangle-\ln \phi(t): t \in \mathbf{R}^{d}\right\} .
$$

If $A \subset \mathbf{R}^{d}$, we set (in this section only)

$$
I(A):=\inf \{I(x): x \in A\} .
$$

Assuming (1.1), the classical rough large deviation estimates assert that for any Borel set $A \subset \mathbf{R}^{d}$ with interior int $A$ and closure $\operatorname{cl} A$,

$$
\begin{aligned}
-I(\operatorname{int} A) & \leqslant \liminf _{n \rightarrow \infty} n^{-1} \ln P\left\{S_{n} \in n A\right\} \\
& \leqslant \limsup _{n \rightarrow \infty} n^{-1} \ln P\left\{S_{n} \in n A\right\} \leqslant-I(\operatorname{cl} A) .
\end{aligned}
$$

The properties of the function $I$ are described in detail by Borovkov and Mogulskii [11], [12]. We refer to the lectures by Azencott [2], the books by Stroock [43], Ellis [27], Deuschel and Stroock [23], Bucklew [19], and Dembo and Zeitouni [22] for introductions or detailed accounts on the theory of rough large deviations and its applications.

Much less is known on precise large deviations. When $d=1$, Bahadur and Rao's [3] or Petrov's [36] formulas deal with the natural sets $[a, \infty)$ and assert that (provided the distribution of $X$ has some mild regularity property)

$$
P\left\{S_{n} \in n[a, \infty)\right\} \sim \frac{e^{-n I(a)}}{\sqrt{n} \psi(a)} \quad \text { as } \quad n \rightarrow \infty,
$$

where $\psi(\cdot)$ is a function which is calculated from the moment generating function $\phi$, and consequently depends only on the distribution of the random variables. Vinogradov [44] also deals with precise large deviation, but is mainly concerned in what happens when (1.1) does not hold.

When $d \geqslant 1$, there is no natural analogue of the intervals $[a, \infty)$, and less was known until recently. However, Borovkov and Rogozin [15] obtained an asymptotic expansion for the density of $S_{n} / n$ in the large deviation domain. Integrating this expansion, they derived one for the probability that $S_{n} / n$ belongs to some well-behaved set $A$. Nagaev [34] obtained some rather precise lower bound when $A$ is a closed set, and a rather precise estimate when $X$ is Gaussian. When $X$ is normal, the distribution of $S_{n}$ is also normal, and techniques of asymptotic analysis allowed Ruben [40] and Breitung and Hohenbichler [16] to obtain an analogue of (1.3), i.e., an equivalent of $P\left\{S_{n} \in n A\right\}$, for sets $A$ having a smooth boundary. In the nongaussian cases, Ney [35] evaluated precise large deviation probabilities for some sets which are cusp like. More recently, Iltis [31] dealt with some sets $A$ which are 
smooth (i.e., the boundary $\partial A$ is a smooth manifold) or conic cusps. In both situations, he takes advantage of an explicit parametrization of the set $\partial A$. Improving upon Iltis [31], Andriani and Baldi [1] obtained an approximation of the density of $S_{n} / n$, uniformly over compact sets. Integrating their approximation, they could recover Iltis's result, but they focus on the geometry of $A$ when $\partial A$ is smooth and has a unique point, where the function $I$ reaches its minimum. Borovkov and Mogulskii [14] obtained an integro-local large deviation result under weaker conditions than the above mentioned authors, but with a slight restriction on the sets $A$ to be considered. In a somewhat different vein, Borovkov and Mogulskii [11]-[13] obtained sharp result under very weak assumptions on the distribution and focusing on uniformity with respect to the distribution and the set. It is not very clear who first obtained an approximation of the density of $S_{n} / n$, and the reader may refer to Jensen [32], where saddlepoint approximations for the density of $S_{n} / n$ are discussed in great detail.

The main aim of this paper is to settle completely the question of precise large deviations for arbitrary Borel set, under the same assumptions as Iltis [31] or Andriani and Baldi [1], but with a different approach. Our results turn to be new even for $d=1$.

To understand the amazing feature of such result, the reader has to be aware of how bad the rough estimate (1.2) can be. A first trivial case is when $A=(\mathbf{R} / \mathbf{Q})^{d}$ or $\mathbf{Q}^{d}$, so that the left-hand side of $(1.2)$ is $-\infty$ while the right-hand side is 0 . A second case is when $A$ is closed and $I(A)=I(x)$ for a unique $x \in A$ which is also isolated. In this case $I(\operatorname{int} A)<I(A)$. A far less trivial example is given in Slaby [41] who exhibits some random variables and an open set $A$ such that

$$
\liminf _{n \rightarrow \infty} n^{-1} \ln P\left\{S_{n} \in n A\right\}>-I(A),
$$

showing that the lower bound in (1.2) may not be sharp at all. He also proves the interesting fact that the upper bound

$$
\limsup _{n \rightarrow \infty} n^{-1} \ln P\left\{S_{n} \in n A\right\} \leqslant-I(A)
$$

holds for any Borel set $A \subset \mathbf{R}^{d}$, provided that there is no affine subspace $J$ of codimension 2 of the affine hull $H$ of the support $S$ of the distribution of $X$ such that

$$
P\left\{X \in J \cap \partial_{H} \operatorname{conv} S\right\}>0 .
$$

However, Bahadur and Zabell [4] showed that for open convex sets $A$ (or finite union of them), then the limsup and lim inf in (1.2) can be replaced by a limit which turns to be equal to $-I(A)$. Of course, the same is trivially true for any set $A$ such that $I(\operatorname{int} A)=I(\operatorname{cl} A)$. 
For any distribution satisfying some smoothness condition (see mainly our assumption (2.2)), our sharp large deviation estimate implies that for any arbitrary Borel set $A$,

$$
\lim _{n \rightarrow \infty} n^{-1} \ln P\left\{S_{n} \in n A\right\}=-I(A)
$$

provided we define $I(A)$ as the essential infimum of $I$ over $A$. This completes Slaby's upper bound.

The reader should be aware that the assumption of independence and identical distribution for the $X_{i}$ 's is not crucial in our proof. The key fact is to be able to obtain good asymptotics for $n^{-1} \ln E \exp \left(i\left\langle t, S_{n}\right\rangle\right)$. This turns to be easy for independent and identically distributed random variables. This could possibly be done for some Markov chain or linear processes.

The organization of the paper is as follows. In the next section we give our main result and some of its consequences. We also state a multidimensional Laplace approximation in the form of an estimate for a probability of large deviation. This main result is proved in Section 3, while its consequences are proved in Section 4. Section 5 contains the proof of our sharp large deviation estimate for lattice distributions. The main result of the paper (Theorem 2.1) is due to both authors. Proposition 2.1 and the geometric construction are due to the first named one.

2. The main result and its consequences. In order to obtain a sharp estimate of $P\left\{S_{n} \in n A\right\}$ we need few assumptions besides the classical (1.1). We denote

$$
m(t):=D \ln \phi(t) \in \mathbf{R}^{d} \quad \text { and } \quad H(t):=\text { Hess } \ln \phi(t)
$$

the gradient and Hessian of $\ln \phi$ evaluated at point $t$. Let $\mathscr{N}$ be the natural parameter space of the standard exponential family $\left(P_{t}\right)_{t \in \mathcal{N}}$ associated with $P$, i.e.,

$$
\mathscr{N}:=\left\{t \in \mathbf{R}^{d}: \phi(t)<\infty\right\}
$$

and

$$
d P_{t}(x):=\frac{e^{\langle t, x\rangle}}{\phi(t)} d P(x)
$$

Let $\mathscr{H}$ be the convex hull of the support of $P$. We shall assume that

$$
m \text { is a homeomorphism from int } \mathscr{N} \text { to int } \mathscr{H} \text {. }
$$

When studying the large deviation probability $P\left\{S_{n} \in n A\right\}$ for some specific set, assumption (2.1) can be weakened considerably and Comment 2.7 below elaborates on this point.

If (2.1) holds, we consider the variance function $V$ (see, e.g., [33]) which is just the expression of $H(t)$ as a function of $m(t)$, so that

$$
V(a):=H\left(m^{\mathrm{inv}}(a)\right)
$$


(here and in what follows the superscript inv means the inverse of a function, while the superscript ${ }^{-1}$ is to indicate the inverse of an element in a group, like the inverse of a matrix; thus, if $M(a)$ is a matrix-valued function, $M\left(M^{\text {inv }}(a)\right)=a$ while $\left.M(a) M^{-1}(a)=\mathrm{Id}\right)$. If $M$ is a matrix we denote by $|M|$ or $\operatorname{det} M$ its determinant.

A sufficient condition for (2.1) to hold is that the family $\left(P_{t}\right)$ is minimal (i.e., $\operatorname{dim} \mathscr{N}=\operatorname{dim} \mathscr{H}$ ) and steep (i.e., $\lim _{t \rightarrow \partial \mathscr{N}}|m(t)|=+\infty$ ) (see, e.g., [6], [17], and [33]). We believe (1.5) is related to some kind of minimality.

Furthermore, let $\widehat{p}_{t}(\lambda)$ be the characteristic function of $P_{t}$, i.e.,

$$
\widehat{p}_{t}(\lambda):=\int e^{i\langle\lambda, x\rangle} d P_{t}(x)=\frac{\int e^{\langle x, i \lambda+t\rangle} d P(x)}{\phi(t)} .
$$

We assume that

$$
\widehat{p}_{t} \in L^{r}\left(\mathbf{R}^{d}, d x\right) \text { for some } r \geqslant 1 \text { and any } t \in \mathscr{N} .
$$

Notice that (2.1) is assumption (1.3) in Iltis [31], and (2.2) is the conclusion of Iltis's Lemma 1.1. Taking $t=0$ in (2.2) yields that the $r$-th convolution $P^{* r}$ is absolutely continuous for some $r$.

Not any distribution satisfies (2.2), but all the usual absolutely continuous (normal, exponential, gamma, etc.) do. Feller [28, Chap. VI, §5] gives examples, where (2.2) does not hold. In such situation, some pathology can occur. In particular (2.2) guarantees that a local central limit theorem holds for random variables with distribution $P_{t}$, and in particular for $P_{0}=P$. Our proof shows that it is really what we need.

We denote $\Lambda_{c}$ the level set

$$
\Lambda_{c}:=\left\{x \in \mathbf{R}^{d}: I(x)=c\right\} .
$$

Also, we define the essential infimum of $I$ on a set $A$ with respect to the Lebesgue measure, ess inf $\{I(a): a \in A\}$, as the infimum of $I(a)$ taken over all $a \in A$ such that for any neighborhood $B$ of $a$, the Lebesgue measure of $B \cap A$ is positive.

Our first result gives an estimate of $P\left\{S_{n} \in n A\right\}$. There is no reason to have an asymptotic equivalent as $n \rightarrow \infty$, and one may have to consider subsequences (which is somewhat hidden in statement of the following theorem but will be clear in the next one). The result is not new, but we believe our proof is. Thus, in some sense, this result is important here mostly for its proof.

Theorem 2.1. Assume (1.1), (2.1), (2.2). Let $A$ be a relatively compact Borel set in $\mathbf{R}^{d}$ with positive Lebesgue measure and such that

$$
I(A):=\operatorname{essinf}\{I(a): a \in A\}<\infty .
$$


Then

$$
P\left\{S_{n} \in n A\right\} \sim\left(\frac{n}{2 \pi}\right)^{d / 2} \int_{A}|V(a)|^{-1 / 2} e^{-n I(a)} d a \quad \text { as } \quad n \rightarrow \infty .
$$

Few comments can be made on Theorem 2.1.

$\mathrm{C}$ o $\mathrm{m} \mathrm{m} \mathrm{e} \mathrm{n} \mathrm{t}$ 2.1. $I(A)$ is defined as an essential infimum (with respect to the Lebesgue measure), and not as an infimum as usual. The assumption that $A$ is of positive Lebesgue measure is not restrictive. If $A$ is Lebesgue negligible, (2.2) yields $P\left\{S_{n} \in n A\right\}=0$ for $n$ large enough, which of course settles trivially the estimation of the precise large deviation.

The strict convexity of $\ln \phi(t)$ implies that

$$
V(a) \text { or equivalently } H(t) \text { are positive definite. }
$$

Therefore $|V(a)|^{-1 / 2}$ in statement of Theorem 2.1 is well defined. However, nothing prevents $|V(a)|$ to degenerate when $a$ tends to $\infty$ along certain path. Therefore, for an unbounded set $A$, the integral $\int_{A}|V(a)|^{-1 / 2} e^{-n I(a)} d a$ could possibly be infinite. Although we did not find any such example, we have not been able to prove that this cannot happen. Thus, for nonrelatively compact set $A$, Theorem 2.1 need to be rephrased. A possible formulation, which is actually a key step in proving Theorem 2.1, is as follows.

Theorem 2.1'. Let $\mu_{A, n}$ be the sequence of probability measures defined by

$$
\mu_{A, n}(B):=\frac{\int_{A \cap B} e^{-n I(a)} d a}{\int_{A} e^{-n I(a)} d a} .
$$

i) The sequence of probability measures $\mu_{A, n}$ is tight.

ii) If $n_{k}$ is a subsequence and $\mu_{A}$ is a measure such that $\mu_{A, n_{k}}$ converges to $\mu_{A}$ as $k \rightarrow \infty$, then $\operatorname{supp} \mu_{A} \subset \Lambda_{I(A)}$. Along such subsequence,

$$
P\left\{S_{n_{k}} \in n_{k} A\right\} \sim\left(\frac{n_{k}}{2 \pi}\right)^{d / 2} \int_{A} e^{-n_{k} I(a)} d a \int_{\Lambda_{I(A)}}|V(a)|^{-1 / 2} d \mu_{A}(a)
$$

as $k \rightarrow \infty$.

$\mathrm{C} \circ \mathrm{m} \mathrm{m}$ e $\mathrm{n} \mathrm{t} 2.2$. The mass of the measure $\mu_{A}$ is necessarily 1 . Thus, if

$$
v_{U}(A):=\sup \left\{|V(a)|: a \in \Lambda_{I(A)}\right\}, \quad v_{L}(A):=\inf \left\{|V(a)|: a \in \Lambda_{I(A)}\right\},
$$

then (2.3) and the compactness of $\Lambda_{I(A)}$ imply

$$
0<v_{U}\left(\Lambda_{I(A)}\right)^{-1 / 2} \leqslant \int|V(a)|^{-1 / 2} d \mu_{A}(a) \leqslant v_{L}\left(\Lambda_{I(A)}\right)^{-1 / 2}<\infty .
$$

Hence, Theorem 2.1 gives indeed a nondegenerated asymptotic equivalent. 
$\mathrm{C} \mathrm{o} \mathrm{m} \mathrm{m}$ e $\mathrm{nt}$ 2.3. Introducing the level set

$$
\Gamma_{c}:=\left\{x \in \mathbf{R}^{d}: I(x) \leqslant c\right\}
$$

we can define the function

$$
L_{A}(t):=\left|A \cap \Gamma_{I(A)+t}\right| .
$$

Then

$$
\int_{A} e^{-n I(a)} d a=e^{-n I(A)} \int e^{-n t} d L_{A}(t) .
$$

It follows that the equivalent of $P\left\{S_{n} \in n A\right\}$ is related to $L_{A}$ by its Laplace transform. Specifically, assertion ii) of Theorem $2.1^{\prime}$ shows that the rate of convergence to 0 of $P\left\{S_{n} \in n A\right\}$ is entirely given by the behavior of $L_{A}(t)$ (but the constant in the equivalent given in ii) depends on $\mu_{A}$ ).

This will allow us to make use of the standard Abel-Tauber theorems for Laplace transform (see, e.g., [7]). This also shows the crucial importance of the function $L_{A}$ for applications.

$\mathrm{C}$ o $\mathrm{m} \mathrm{m}$ e $\mathrm{nt}$ 2.4. In Theorem 2.1 the relative compactness assumption is mainly to ensure that the integral is finite. If $A$ is not relatively compact, on could replace the integral over $A$ by an integral over $A \cap \Gamma_{I(A)+1}$ say.

$\mathrm{C}$ o $\mathrm{m} \mathrm{m} \mathrm{e} \mathrm{n} \mathrm{t} \mathrm{2.5.} \mathrm{When} d=1$, the set $\Lambda_{I(A)}$ contains at most two points. Hence, if $A \subset(E X, \infty)$ or $A \subset(-\infty, E X)$, the measure $\mu_{A}$ is just the Dirac measure $\delta_{I(A)}$.

C o m m e n t 2.6. Assumption (2.3) with $t=0$ ensures that $S_{n} / n$ has a density $f_{S_{n} / n}$ for $n$ large enough. The statement in Theorem 2.1 can be rewritten

$$
\int_{A} f_{S_{n} / n}(a) d a \sim \int_{A}\left(\frac{n}{2 \pi}\right)^{d / 2}|V(a)|^{-1 / 2} e^{-n I(a)} d a,
$$

showing a weak approximation of the density $f_{S_{n} / n}(a)$. An approximation in a much stronger sense, but in the range $a=o(1)$ is given by Richter in [38] in the one-dimensional case, and in [39] in a multidimensional setting. Iltis [31] gives the approximation of the density for a fixed $a$ in his Corollary 1.1, restating a previous result of Borovkov and Rogozin [15] and Chaganty and Sethuraman [20]. Jensen [32] and Andriani and Baldi [1] proved the approximation of the density over fixed compact sets. Therefore our Theorem 2.1 could be viewed as a consequence of Borovkov and Rogozin's, Jensen's or Andriani and Baldi's results. However the interesting feature of our result is in its proof, somewhat different than the standard ones. Theorem $2.1^{\prime}$ is new. We shall obtain in what follows some more complete results than Andriani and Baldi [1], which therefore could alternatively be obtained from their main theorems. Notice that Borovkov and Mogulskii [14, Part I] give an expression for $P\left\{S_{n} \in n A\right\}$ under a condition weaker than (2.2), namely 
assuming that $\lim \sup _{|s| \rightarrow \infty}|\phi(i s)|<1$. The counterpart of this weaker assumption shows that the set $A$ needs not only to be measurable but also have a well-behaved boundary (see [14, Part I, p. 4]).

C o m m e n t 2.7. As noticed in [14, Part I], if $A$ is fixed, assumption (2.1) can be weakened considerably. Indeed, it suffices to assume that for some positive $\varepsilon, m\left(\right.$ int $\mathscr{N}$ ) contains $A \cap \Lambda_{I(A)+\varepsilon}$. Indeed, the usual rough estimate (1.2) shows that $P\left\{S_{n} \in n A\right\} \sim P\left\{S_{n} \in n\left(A \cap \Lambda_{I(A)+\varepsilon}\right)\right\}$ as $n$ tends to infinity.

As pointed out in the introduction, it is known that the large deviation bound (1.2) may not be sharp. The following corollary settles definitely the question under the assumptions of Theorem 2.1. It provides an amazingly simple answer, which seems to be new even for $d=1$.

Corollary 2.1. Under the assumptions of Theorem 2.1, for any Borel set $A \subset \mathbf{R}^{d}$,

$$
\lim _{n \rightarrow \infty} n^{-1} \ln P\left\{S_{n} \in n A\right\}=-I(A) .
$$

Recall that $I(A)$ is defined in Theorem 2.1 with the essential infimum, and not the usual infimum. What Corollary 2.1 tells us is that the sharpness of (1.2) depends mainly on the smoothness of the distribution. This somewhat gives some insight into Slaby's [41] example. His example works thanks to his very clever choice of a somewhat pathological distribution.

In the cases covered in Iltis [31], one can easily estimate the function $L_{A}(t)$ (or at least, and this is what matters here, give an asymptotic estimate as $t \rightarrow 0$ ), show that the sequence $\mu_{A, n}$ converges, and recover his results. The following characterizes all the situations, such as those handled by Iltis [31], where $P\left\{S_{n} \in n A\right\}$ has a leading term $e^{-n I(A)}$ corrected by some power of $n$. This is nothing but a restatement of Karamata's Tauberian theorem. Recall that a function $\ell$ is called slowly varying at infinity if

$$
\text { for any } \quad \lambda>0, \quad \lim _{x \rightarrow \infty} \frac{\ell(\lambda x)}{\ell(x)}=1
$$

which is denoted by $\ell \in R_{0}(\infty)$.

Corollary 2.2. The following are equivalent (where $\ell \in R_{0}(\infty)$ ):

i) $L_{A}(t) \sim t^{\rho} \ell(1 / t) / \Gamma(1+\rho)$ as $t \rightarrow 0$,

ii) $\int_{A} e^{-n I(a)} d a \sim e^{-n I(A)} n^{-\rho} \ell(n)$ as $n \rightarrow \infty$.

It can of course happen that $L_{A}(t)$ tends to 0 faster than any power of $t$ and at exponential rate. In this case we may end up having an equivalent of the form $\int_{A} e^{-n I(a)} d a \sim \alpha_{1} e^{-n I(A)-\alpha n^{\rho}}$ for some $\rho<1$. The relevant Tauberian result is due to de Bruijn [18] (see [7, Theorem 4.12.9]); it gives only an estimate of $\ln \left(\int_{A} e^{-n I(a)} d a\right)+n I(A)$ in terms of $\ln L_{A}(t)$. More precise estimates are in [45] and [5]. 
Corollary 2.3. Let $\alpha>0$. Then

$$
-\ln L_{A}(t) \sim t^{-1 / \alpha} \ell_{1}(t) \quad \text { as } \quad t \rightarrow 0,
$$

for some function $\ell_{1} \in R_{0}(\infty)$, if and only if

$$
\ln \left(\int_{A} e^{-n I(a)} d a\right)+n I(A) \sim n^{-1 /(\alpha+1)} \ell_{2}(n),
$$

for some function $\ell_{2} \in R_{0}(\infty)$.

Of course the functions $\ell_{1}$ and $\ell_{2}$ in Corollary 2.3 are related to each others, but the exact relation (see, e.g., [7, Theorem 4.12.9]) does not really bring much insight here.

We now turn to a result more in the spirit of Iltis [31]. Iltis [31] discusses mainly large deviation probabilities for some sets $A$ with a smooth boundary $\partial A$, and he makes use of an explicit parametrization of the set $\partial A$. In our opinion, and this was also pointed out by Andriani and Baldi [1], this makes the problem of finding equivalent of $P\left\{S_{n} \in n A\right\}$ very analytic and does not show much how the geometry of the set comes into play. The purpose of the following is to show that differential geometric quantities do govern the large deviation behavior of $S_{n} / n$ in smooth sets.

Let us assume now that

\section{$\partial A$ is a smooth manifold}

(here smooth means at least $C^{3}$ ). Let $c=I(A)$ and assume that $A$ has a $k$-dimensional manifold of dominating-points, that is,

$$
D=\partial A \cap \Lambda_{c} \text { is a } k \text {-dimensional manifold. }
$$

If $k=0$ it is clear that $D$ reduces to a finite number of points, and there is no loss of generality in assuming that $D$ is reduced to a single point. This case $(k=0)$ has been studied by Borovkov and Rogozin [15], Borovkov and Mogulskii [11], [12], Iltis [31] as well as Andriani and Baldi [1]. It is remarkable that Borovkov and Mogulskii [14] also settled the case $k=0$ under assumptions on the distribution that are weaker than those used by Iltis [31], Andriani and Baldi [1] and the current paper, but for a slightly different class of sets. When $k=0$, the asymptotic approximation of $P\left\{S_{n} \in n A\right\}$ can be deduced from saddlepoint approximations and expansion of Laplace integrals as in Section 8.3 of Bleistein and Handelsman [8]. Notice that $D$ may be a manifold with boundary, since it is a closed subset of $\Lambda_{c}$. The natural Riemannian structure on $\mathbf{R}^{d}$ makes $D$ a Riemannian manifold. We need the following technical assumption:

if $k>0$, there exists a $k$-dimensional manifold (without boundary)

$$
\mathscr{D} \subset \Lambda_{c} \text { which contains } D \text {. }
$$


If $D$ has no boundary, we can just consider $\mathscr{D}=D$. If $D$ as a boundary $\partial D$ and if at each point $p \in \partial D$ we can define half a tangent space, then we can define $\mathscr{D}$ in adding to $D$ the image of small vectors in the half tangent space by the exponential map (on $\Lambda_{c}$ ) at any point of $\partial D$. But this is not always possible (think for instance of extending this way the curve $\{(x, x \sin 1 / x): x \geqslant 0\}$ of $\left.\mathbf{R}^{2}\right)$.

We denote $\mathscr{M}$ the Riemannian measure on $D$, which can be extended to $\mathscr{D}$ (if $k=0$ and $D=\{p\}$ we set $\mathscr{D}=D$ and $\mathscr{M}=\delta_{p}$ ). Nothing prevents $\mathscr{D}$ to be of infinite $\mathscr{M}$ measure, and we assume that

$$
\mathscr{M}(\mathscr{D}) \text { is finite. }
$$

We have the immersions $\mathscr{D} \subset \Lambda_{c} \subset \mathbf{R}^{d}$. For each $p \in \mathscr{D}$, we can split $\mathbf{R}^{d}$ into the orthogonal sum $T_{p} \mathscr{D} \oplus \widetilde{N}_{p} \mathscr{D} \oplus N_{p} \Lambda_{c}$, where $T_{p} \mathscr{D}$ is the $k$-dimensional tangent fiber of $\mathscr{D}$ at $p, \tilde{N}_{p} \mathscr{D}$ is the $(d-k-1)$-dimensional normal fiber of $\mathscr{D}$ (at $p$ ) immersed in $\Lambda_{c}$, and $N_{p} \Lambda_{c}$ is the normal fiber of $\Lambda_{c}$ (at $p$ ) immersed in $\mathbf{R}^{d}$. Then $N_{p} \mathscr{D}:=\widetilde{N}_{p} \mathscr{D} \oplus N_{p} \Lambda_{c}$ is the normal fiber of $\mathscr{D}$ immersed in $\mathbf{R}^{d}$. Now let $\mathscr{S}_{p} \partial A$ and $\mathscr{S}_{p} \Lambda_{c}$ be the sections of $\partial A$ and $\Lambda_{c}$ along $N_{p} \mathscr{D}$, i.e. (in the space $\left.\mathbf{R}^{d}\right) \mathscr{S}_{p} \partial A:=A \cap N_{p} \mathscr{D}$ and $\mathscr{S}_{p} \Lambda_{c}=\Lambda_{c} \cap N_{p} \mathscr{D}$. Then $\mathscr{S}_{p} \partial A$ and $\mathscr{S}_{p} \Lambda_{c}$ are $(d-k-1)$-dimensional submanifolds with common tangent fiber $N_{p} D$ at $p \in D$. Let $\mathscr{W}_{\mathscr{S}_{p} \partial A, p}$ and $\mathscr{W}_{\mathscr{S}_{p} \Lambda_{c}, p}$ be the Weingarten maps at point $p$ associated with $\mathscr{S}_{p} \partial A$ and $\mathscr{S}_{p} \Lambda_{c}$ as submanifolds of $\mathbf{R}^{d}$ and the normal direction $N_{p} \Lambda_{c}$ (we choose here the unit normal in $N_{p} \Lambda_{c}$ pointing outward $\left.\Gamma_{c}\right)$, i.e., $x \in \widetilde{N}_{p} D \equiv T_{p}\left(\mathscr{S}_{p} \partial A\right) \equiv T_{p}\left(\mathscr{S}_{p} \Lambda_{c}\right) \mapsto\left\langle\mathscr{W}_{M, p} x, x\right\rangle$ is the second fundamental form of $\mathscr{S}_{p} M$ for $M=\mathscr{S}_{p} \partial A, \mathscr{S}_{p} \Lambda_{c}$.

Recall that $G_{M}(p):=\operatorname{det} \mathscr{W}_{M, p}$ is the Gauss-Kronecker curvature of $M$ at $p$ for $M=\mathscr{S}_{p} \partial A, \mathscr{S}_{p} \Lambda_{c}$. Now, imagine we are living on $\mathscr{S}_{p} \Lambda_{c}$ and look at the manifold $\mathscr{S}_{p} \partial A$. For us, the vertical at a point $p$ is the normal direction to $\mathscr{S}_{p} \Lambda_{c}$, and $\mathscr{S}_{p} \partial A$ has a Gauss-Kronecker curvature given by $G_{\mathscr{S}_{p} \Lambda_{c}, \mathscr{S}_{p} \partial A}(p):=\operatorname{det}\left(\mathscr{W}_{\mathscr{S}_{p} \partial A, p}-\mathscr{W}_{\mathscr{S}_{p} \Lambda_{c}, p}\right)$. We shall assume that

$$
\begin{gathered}
\mathscr{W}_{\mathscr{S}_{p} \partial A, p}-\mathscr{W}_{\mathscr{S}_{p} \Lambda_{c}, p} \quad \text { is definite positive on } \\
\tilde{N}_{p} D=T_{p}\left(\mathscr{S}_{p} \Lambda_{c}\right)=T_{p}\left(\mathscr{S}_{p} \partial A\right) .
\end{gathered}
$$

Consequently, $G_{\mathscr{S}_{p} \Lambda_{c}, \mathscr{S}_{p} \partial A}(p)>0$.

Proposition 2.1. Under the hypotheses of Theorem 2.1 and (2.4)-(2.8),

$$
\begin{aligned}
P\left\{S_{n} \in n A\right\} \sim & e^{-n I(A)} n^{(k-1) / 2}(2 \pi)^{-(k+1) / 2} \\
& \times \int_{D} \frac{d \mathscr{M}(p)}{|V(p)|^{1 / 2}\left|m^{\text {inv }}(p)\right|^{(d-k+1) / 2} G_{\mathscr{S}_{p} \Lambda_{c}, \mathscr{S}_{p} \partial A}(p)^{1 / 2}} .
\end{aligned}
$$

When $k=d-1, \tilde{N}_{p} D$ is empty and one has to $\operatorname{read} G_{\Lambda_{c}, \partial A}(p)=1$ in Proposition 2.1. 
As expected, Proposition 2.1 tells us that a set $A$ with higherdimensional dominant manifold (i.e., $k$ large) has higher probability, as well as a set $A$ which does not pull away too fast from its dominating manifold (i.e., for which $G_{\mathscr{S}_{p} \Lambda_{c}, \mathscr{S}_{p} \partial A}$ is small), as well as a set $A$ with large dominating manifold (i.e., $\mathscr{M}(D)$ is large).

Proposition 2.1 contains Theorems 1.4 and 1.3 of [31] as special cases, as well as the result of [1]. It also extends some classical approximation of multidimensional integral of Laplace type (see, e.g., $[8, \S 8.3]$ ).

Indeed, substituting $|V(\cdot)|^{1 / 2}$ by a function $\psi(\cdot)$ say, it asserts (and its proof shows) that

$$
\begin{aligned}
\int_{A} \psi(a) e^{-n I(a)} d a \sim & e^{-n I(A)} n^{(k-1-d) / 2}(2 \pi)^{(k-1-d) / 2} \\
& \times \int_{D} \frac{\psi d \mathscr{M}}{|D I|^{(d-k+1) / 2} G_{\mathscr{S}_{p} \Lambda_{c}, \mathscr{S}_{p} \partial A}(p)^{1 / 2}}
\end{aligned}
$$

as $n$ tends to infinity, provided $\psi$ is smooth and bounded say, and that $I$ is a strictly convex function say, with its minimum on the boundary of $A$.

Another use of Theorem 2.1 and Proposition 2.1 is to provide information on some conditional distributions of $S_{n} / n$. The idea is as follows. Consider two Borel sets $B \subset A \subset \mathbf{R}^{d}$. Theorem 2.1 intuitively tells us that for $n$ large,

$$
\begin{aligned}
P\left(\frac{S_{n}}{n} \in B \mid \frac{S_{n}}{n} \in A\right) & \approx \frac{\int_{A \cap B}|V(a)|^{-1 / 2} e^{-n I(a)} d a}{\int_{A}|V(a)|^{-1 / 2} e^{-n I(a)} d a} \\
& =\frac{\int_{A \cap B}|V(a)|^{-1 / 2} d \mu_{A, n}(a)}{\int_{A}|V(a)|^{-1 / 2} d \mu_{A, n}(a)} \approx \frac{\int_{B}|V(a)|^{-1 / 2} d \mu_{A}(a)}{\int_{A}|V(a)|^{-1 / 2} d \mu_{A}(a)}
\end{aligned}
$$

Thus one can expect the distribution of $S_{n} / n$ given $S_{n} / n \in A$ to converge weakly* to the measure which puts mass 1 on $A$ and is proportional to $|V(a)|^{-1 / 2} d \mu_{A}$. This can be made rigorous in many cases, and we consider here the interesting one, where $A=\Gamma_{c}^{c}$. This tells us which are the points to be the most likely occupied by $S_{n} / n$ if we know that $I\left(S_{n} / n\right) \geqslant c$.

Corollary 2.4. Under the assumptions of Theorem 2.1, the distribution of $S_{n} / n$ given $I\left(S_{n} / n\right) \geqslant c$ converges weakly* to the probability measure

$$
\frac{\left|m^{\text {inv }}(p)\right|^{-1}|V(p)|^{-1 / 2} d \mathscr{M}(p)}{\int_{\Lambda_{c}}\left|m^{\text {inv }}(p)\right|^{-1}|V(p)|^{-1 / 2} d \mathscr{M}(p)},
$$

where $\mathscr{M}$ is the Riemannian measure on $\Lambda_{c}$.

Corollary 2.4 shows also that it is somewhat more natural to work in $m^{\text {inv }}(\mathscr{H})$. In this case, if $\mathscr{M}^{*}$ is the Riemannian measure on $m^{\text {inv }}\left(\Lambda_{c}\right)$, Corollary 2.4 tells us that $m^{\text {inv }}\left(S_{n} / n\right)$ given $I\left(S_{n} / n\right) \geqslant c$ has the limiting distribution

$$
\nu(d t)=\frac{|t|^{-1} d \mathscr{M}^{*}(t)}{\int_{m^{\text {inv }}\left(\Lambda_{c}\right)}|t|^{-1} d \mathscr{M}^{*}(t)} .
$$


Using either Borovkov and Rogozin's [15], Borovkov and Mogulskii's [11], [12] or Andriani and Baldi's [1] approximation of $f_{S_{n} / n}$, we can obtain a more precise result, namely that $m^{\text {inv }}\left(S_{n} / n\right)$ given $I\left(S_{n} / n\right)=c$ has limiting distribution $\nu(d t)$.

Our final example shows that for nonregular sets, it can be needed to consider subsequences in Theorem 2.1 if one wants to go further than its general statement and do a concrete computation.

Take $d=1$ and to make things easy, assume that $X_{i}$ has a standard normal distribution. Thus $I(x)=x^{2} / 2$ and $V(x)=1$. Let $c>0$, and let further

$$
I:=\left[\frac{1}{3}, \frac{1}{2}\right] \bigcup\left[\frac{3}{4}, 1\right] \text { and } J:=\left[\frac{3}{8}, \frac{1}{2}\right] \bigcup\left[\frac{2}{3}, 1\right] \text {. }
$$

Next, set

$$
A:=\bigcup_{n \in \mathbf{Z}} 4^{n} I \quad \text { and } \quad B=\bigcup_{n \in \mathbf{Z}} 4^{n} J .
$$

Then $4^{n} A=A$ and $4^{n} B=B$ for any $n \in \mathbf{Z}$, while $2 A=B \neq A$.

Applying ii) of Theorem $2.1^{\prime}$ we obtain, as $n \rightarrow \infty$,

$$
\begin{aligned}
P\left\{S_{n} \in n(A+c)\right\} & \sim\left(\frac{n}{2 \pi}\right)^{1 / 2} \int_{A+c} e^{-n x^{2} / 2} d x \sim\left(\frac{n}{2 \pi}\right)^{1 / 2} \int_{A} e^{-n(y+c)^{2} / 2} d y \\
& \sim\left(\frac{n}{2 \pi}\right)^{1 / 2} e^{-n c^{2} / 2} \int_{A} e^{-n\left(c y+y^{2} / 2\right)} d y \sim \frac{e^{-n c^{2} / 2}}{\sqrt{2 \pi n}} \int_{n A} e^{-c z} d z .
\end{aligned}
$$

Therefore

$$
\begin{array}{ll}
P\left\{S_{n} \in n(A+c)\right\} \sim \frac{e^{-n c^{2} / 2}}{\sqrt{2 \pi n}} \int_{A} e^{-c z} d z \quad \text { for } n=4^{k}, \quad k \rightarrow \infty, \quad \text { and } \\
P\left\{S_{n} \in n(A+c)\right\} \sim \frac{e^{-n c^{2} / 2}}{\sqrt{2 \pi n}} \int_{B} e^{-c z} d z \quad \text { for } n=2 \cdot 4^{k}, \quad k \rightarrow \infty .
\end{array}
$$

We now provide an example, where the sequence of measures $\mu_{A, n}$ in Theorem 2.1' does not converge. Let $d=2$ and let $X_{i}=\left(X_{1, i}, X_{2, i}\right)$ have a normal distribution $\mathscr{N}(0, \mathrm{Id})$. Let $c>0$. Let $B_{1}:=\bigcup_{k \in \mathbf{Z}} 4^{k}\left[\frac{1}{2}, 1\right]$ and $B_{2}:=\bigcup_{k \in \mathbf{Z}} 4^{k}[1,2]$, so that $B_{1} \cup B_{2}=(0, \infty)$. Let $C_{1}$ (respectively, $C_{2}$ ) be the set of all points in $\mathbf{R}^{2}$ with polar coordinates $(r, \theta)$ such that $0 \leqslant \theta \leqslant \pi / 6$ (respectively, $\pi / 3 \leqslant \theta \leqslant \pi / 2$ ) and $r \in B_{1}+c$ (respectively, $r \in B_{2}+c$ ). Finally, let $A:=C_{1} \cup C_{2}$. Then, as $n \rightarrow \infty$,

$$
\begin{aligned}
\int_{A} e^{-n I(a)} d a= & \int_{r \in B_{1}+c} \int_{\theta \in[0, \pi / 6]} e^{-n r^{2} / 2} r d r d \theta \\
& +\int_{r \in B_{2}+c} \int_{\theta \in[\pi / 3, \pi / 2]} e^{-n r^{2} / 2} r d r d \theta \\
= & \frac{\pi}{6} \int_{r \geqslant c} e^{-n r^{2} / 2} r d r \sim \frac{\pi}{6} \frac{e^{-n c^{2} / 2}}{n} .
\end{aligned}
$$


Furthermore,

$$
\begin{aligned}
\int_{A \cap C_{1}} e^{-n I(a)} d a & =\int_{r \in B_{1}+c} \int_{\theta \in[0, \pi / 6]} e^{-n r^{2} / 2} r d r d \theta \\
& =\frac{\pi}{6} \int_{r \in B_{1}} e^{-n(r+c)^{2} / 2}(r+c) d r \sim \frac{\pi}{6} e^{-n c^{2} / 2} \int_{B_{1}} e^{-n r c} d r .
\end{aligned}
$$

Consequently, as $k \rightarrow \infty$,

$$
\mu_{A, 4^{k}}\left(C_{1}\right) \sim \frac{1}{4^{k}} \int_{B_{1}} e^{-4^{k} r c} d r=\int_{B_{1}} e^{-r c} d r
$$

which is different from

$$
\mu_{A, 2 \cdot 4^{k}}\left(C_{1}\right) \sim \frac{1}{2 \cdot 4^{k}} \int_{B_{1}} e^{-2 \cdot 4^{k} r c} d r=\int_{B_{2}} e^{-r c} d r .
$$

Thus, $\mu_{A, n}$ does not converge.

We now state the analogue of Theorem 2.1 for lattice distributions. We denote by $h=\left(h_{1}, \ldots, h_{d}\right)$ the span of the lattice distribution. If $x, y \in \mathbf{R}^{d}$ we denote by $x y$ the vector $\left(x_{1} y_{1}, \ldots, x_{d} y_{d}\right)$ so that the support of $P$ is contained in $\mathbf{Z}^{d} h=\left\{x h: x \in \mathbf{Z}^{d}\right\}$.

Theorem 2.2. Assume that $X$ has a lattice distribution and that (1.1) and (2.1) hold. Let $A$ be a Borel subset of $\mathbf{R}^{d}$ and set $c_{n}:=$ $\min \{I(k h / n): k h / n \in A\}$. Define the probability measures

$$
\mu_{A, n}(B):=\frac{\sum_{k \in \mathbf{Z}^{d}} 1\{k h / n \in A \cap B\} e^{-I(k h / n)}}{\sum_{k \in \mathbf{Z}^{d}} 1\{k h / n \in A\} e^{-n I(k h / n)}}
$$

if $\mathbf{Z}^{d} h \cap A \neq \varnothing$ (otherwise $P\left\{S_{n} \in n A\right\}=0$ ). Let $c$ be a finite cluster point of the sequence $c_{n}$ and $n_{k}=n_{k}(c)$ such that $\lim _{k \rightarrow \infty} c_{n_{k}}=c$.

i) The sequence $\mu_{A, n_{k}}$ is tight.

ii) If $\mu_{A, n_{k}^{\prime}}$ is a (sub-)subsequence converging to a limiting measure $\mu_{A}$, then $\operatorname{supp} \mu_{A} \subset \Lambda_{c}$. Along such a (sub-) subsequence,

$$
P\left\{S_{n_{k}^{\prime}} \in n_{k}^{\prime} A\right\} \sim\left(\frac{n_{k}^{\prime}}{2 \pi}\right)^{d / 2} \int_{A} 1\left\{I(a) \geqslant c_{n_{k}^{\prime}}\right\} e^{-n_{k}^{\prime} I(a)} d a \int_{\Lambda_{c}}|V(a)|^{-1 / 2} d \mu_{A}(a)
$$

as $n_{k}^{\prime} \rightarrow \infty$.

iii) Moreover, if $A$ is relatively compact, then

$$
P\left\{S_{n} \in n A\right\} \sim\left(\frac{n}{2 \pi}\right)^{d / 2} \int_{A} 1\left\{I(a) \geqslant c_{n}\right\}|V(a)|^{-1 / 2} e^{-n I(a)} d a
$$

as $n \rightarrow \infty$.

The analogue of Corollary 2.1 is as follows. 
Corollary 2.5. If $X_{i}$ has a lattice distribution satisfying (1.1) and (2.1), for any Borel set $A \subset \mathbf{R}^{d}$ and any subsequence $n_{k}$, the limiting points of $n_{k}^{-1} \ln P\left\{S_{n_{k}} \in n_{k} A\right\}$ coincide with those of the sequence $\inf \left\{I(x): x \in n_{k} A \cap \mathbf{Z}^{d} h\right\}$.

Over the last 20 years, motivated by some questions in statistical physics, one has been studying asymptotics for $n^{-1} \ln E \exp \left(n F\left(S_{n} / n\right)\right)$ for some functions $F$. In particular, letting $H_{F}=I-F$, Donsker and Varadhan [26] proved that provided $F$ is smooth enough and does not grow too fast at infinity,

$$
\lim _{n \rightarrow \infty} n^{-1} \ln E \exp \left(n F\left(n^{-1} S_{n}\right)\right)=-\inf _{x \in \mathbf{R}^{d}} H_{F}(x) .
$$

This result has been extended to Banach space by Bolthausen [9], [10]. Our technique of proof yields a much more precise result on $\mathbf{R}^{d}$, with nearly minimal assumptions on $F$.

We shall assume that

$$
\inf _{x \in \mathbf{R}^{d}} H_{F}(x)>-\infty .
$$

Denoting by $u$ this infimum, notice that

$$
n^{-1} \ln E \exp \left(n F\left(n^{-1} S_{n}\right)\right)=n^{-1} \ln E \exp \left(n\left(F\left(n^{-1} S_{n}\right)-u\right)\right)+u
$$

and therefore, there is no loss of generality in what follows to assume that

$$
\inf _{x \in \mathbf{R}^{d}} H_{F}(x)=0
$$

Furthermore, let us suppose that there exists $\varepsilon>0$ such that

$$
\left\{x \in \mathbf{R}^{d}: H_{F}(x) \leqslant \varepsilon\right\} \quad \text { is compact. }
$$

Define the sequence of probability measures

$$
\mu_{F, n}(B)=\frac{\int_{B} e^{-n H_{F}(x)} d x}{\int e^{-n H_{F}(x)} d x},
$$

which are well defined as soon as

$$
\int e^{-n H_{F}(x)} d x<\infty \quad \text { for } n \text { large enough. }
$$

Theorem 2.3. Assume that (1.1), (2.1), (2.2), (2.9)-(2.11) hold. Then

i) the sequence of probability measures $\mu_{F, n}, n \geqslant 1$, is tight;

ii) if $n_{k}$ is a sequence of integers and $\mu$ is a measure such that $\mu_{F, n_{k}}$ converges weakly* to $\mu$ as $k \rightarrow \infty$, then $\operatorname{supp} \mu \subset\left\{x: H_{F}(x)=0\right\}$. Along such a subsequence,

$$
E \exp \left(n_{k} F\left(\frac{S_{n_{k}}}{n_{k}}\right)\right) \sim\left(\frac{n_{k}}{2 \pi}\right)^{d / 2} \int e^{-n_{k} H_{F}(x)} d x \int|V(x)|^{-1 / 2} d \mu(x) .
$$


It should be clear to the reader that Comments 2.1-2.4 can be adapted to the statement of Theorem 2.3. In particular, Comment 2.3 can be written

$$
\int e^{-n H_{F}(x)} d x=\int e^{-n t} d L_{H}(t)
$$

where $L_{H}(t)=\left|\left\{x \in \mathbf{R}^{d}: H_{F}(x) \leqslant t\right\}\right|$. This allows us to obtain asymptotics through Tauberian theorems, and relates the rate of convergence of $n^{-1} \ln E \exp \left(n F\left(S_{n} / n\right)\right)$ to $-\inf _{x} H_{F}(x)$ to the Lebesgue measure of the level sets of $H_{F}$.

One can also see Hwang's [30] results to study the sequence of measures $\mu_{F, n}$.

3. Proofs of Theorem 1.1 and Corollary 2.1. The proof requires few facts which we state as lemmas.

Lemma 3.1. The integral $\int_{\mathbf{R}^{d}} \exp (-I(x)) d x$ converges.

P r o o f. It is enough to show that $\int_{\left(\mathbf{R}^{+}\right)^{d}} \exp (-I(x)) d x$ is finite since the integral over $\prod_{i=1}^{d} \mathbf{R}^{s_{i}}, s_{i}=+,-$, can be handled in the same way. Let $e^{1}, \ldots, e^{d}$ be the canonical basis of $\mathbf{R}^{d}$, and let $t=\eta\left(e^{1}+\cdots+e^{d}\right)$, where $\eta$ is small enough so that $\phi(t)$ is defined (recall assumption (1.1)). The very definition of $I(\cdot)$ yields

$$
\begin{aligned}
\int_{\left(\mathbf{R}^{+}\right)^{d}} \exp (-I(x)) d x & \leqslant \phi(t) \int_{\left(\mathbf{R}^{+}\right)^{d}} \exp (-\langle t, x\rangle) d x \\
& =\phi(t) \int_{c \geqslant 0} \exp (-|t| c) L(c) d c
\end{aligned}
$$

where $L(c)$ is the $(d-1)$-dimensional Lebesgue measure of the simplex

$$
\left\{x \in\left(\mathbf{R}^{+}\right)^{d}:\left\langle\frac{t}{|t|}, x\right\rangle=c\right\} \text {. }
$$

Clearly, $L(c)$ grows as a polynomial in $c$, which yields the result.

Our following lemma proves assertion i) of Theorem 2.1'.

Lemma 3.2. If $|A|>0$, then the sequence of measures $\mu_{A, n}$ is tight, and any limiting measure is concentrated on the set $\Lambda_{I(A)}$.

$\mathrm{Pr}$ o of. The proof is inspired by that of Proposition 2.3 in [30] but with no compactness assumption analogous to Hwang's [30] hypothesis (A.4). Recall that for any $c \geqslant 0$, the level set $\Gamma_{c}$ is compact (see, e.g., [23]). Let $c=\operatorname{essinf}\{I(a): a \in A\}$. First, if $c=\operatorname{ess} \sup \{I(a): a \in A\}$, then the measures $\mu_{A, n}$ are concentrated on the compact set $\Lambda_{c}$, and the result is obvious. Hence, we assume that there exists $c<\alpha \leqslant \operatorname{ess} \sup \{I(a): a \in A\}$, and set $b=(c+\alpha) / 2$ and $\varepsilon$ such that $c+\varepsilon<b$. Thein

$$
\begin{aligned}
\mu_{A, n}\left(\Gamma_{\alpha}^{c}\right) & \leqslant e^{-n b} \frac{\int_{A} 1\{I(a) \geqslant \alpha\} e^{-n(I(a)-b)} d a}{e^{-n(c+\varepsilon)}\left|A \cap \Gamma_{c+\varepsilon}\right|} \\
& \leqslant e^{-n(\alpha-c-2 \varepsilon) / 2} \frac{\int_{A} 1\{I(a) \geqslant \alpha\} e^{-n(I(a)-b)} d a}{\left|A \cap \Gamma_{c+\varepsilon}\right|}
\end{aligned}
$$


(notice that by the very definition of $\left.c,\left|A \cap \Gamma_{c+\varepsilon}\right|>0\right)$. Let $\eta=1-(b / \alpha)=$ $(c+\alpha) /(2 \alpha)$. If $I(a) \geqslant \alpha$, we have $I(a)-b \geqslant \eta I(a)$, and consequently,

$$
\begin{aligned}
\mu_{A, n}\left(\Gamma_{d}^{c}\right) & \leqslant \exp \left(-\frac{n(\alpha-c-2 \varepsilon}{2}\right) \frac{\int_{A} 1\{I(a) \geqslant \alpha\} e^{-n \eta I(a)} d a}{\left|A \cap \Gamma_{c+\varepsilon}\right|} \\
& \leqslant \exp \left(-\frac{n(\alpha-c-2 \varepsilon)}{2}\right) \frac{\int e^{-n \eta I(a)} d a}{\left|A \cap \Gamma_{c+\varepsilon}\right|} .
\end{aligned}
$$

Thus, $\lim _{n \rightarrow \infty} \mu_{A, n}\left(\Gamma_{\alpha}^{c}\right)=0$, for any $\alpha>c$, which proves both the tightness and that any limiting measure is concentrated on $\Lambda_{A}$.

The crucial tool of the proof is the following easy lemma, which somewhat constructs a twisted distribution at any point of the set $A$ !

Lemma 3.3. The following equality holds true:

$$
\iint e^{\left\langle m^{\mathrm{inv}}((s-x) / n), x\right\rangle} 1\left\{\frac{s-x}{n} \in A\right\} d P\left\{S_{n}=s\right\} d x=n^{d} \int_{A} e^{-n I(a)} d a .
$$

$\mathrm{P} \mathrm{r}$ o o f. It suffices to perform the change of variable $a=(s-x) / n$ to obtain that the double integral in Lemma 3.3 is

$$
\begin{aligned}
& \iint e^{\left\langle m^{\mathrm{inv}}(a), s-n a\right\rangle} 1\{a \in A\} d P\left\{S_{n}=s\right\} n^{d} d a \\
& =n^{d} \int \phi\left(m^{\mathrm{inv}}(a)\right)^{n} 1\{a \in A\} e^{-n\left\langle a, m^{\mathrm{inv}}(a)\right\rangle} d a .
\end{aligned}
$$

Now, notice that

$$
I(a)=\left\langle a, m^{\mathrm{inv}}(a)\right\rangle-\ln \phi\left(m^{\mathrm{inv}}(a)\right) .
$$

Lemma 3.3 is proved.

It follows from Lemma 3.3 that for any Borel set $A$ of positive Lebesgue measure, we can define a density

$$
g_{n}(x)=\frac{\int e^{\left\langle m^{\mathrm{inv}}((s-x) / n), x\right\rangle} 1\{(s-x) / n \in A\} d P\left\{S_{n}=s\right\}}{n^{d} \int_{A} e^{-n I(a)} d a},
$$

which has the crucial property that

$$
g_{n}(0)=\frac{P\left\{S_{n} \in n A\right\}}{n^{d} \int_{A} e^{-n I(a)} d a} .
$$

Therefore, the evaluation of $P\left\{S_{n} \in n A\right\}$ reduces essentially to that of $g_{n}(0)$. The relevant technique is that of local central limit theorems, or central limit theorems for density. However, we need to renormalize $g_{n}$ first, and calculate its Fourier transform. 
Lemma 3.4. The characteristic function of the density $h_{n}(x)=$ $n^{d / 2} g_{n}(\sqrt{n} x)$ is

$$
\widehat{h}_{n}(\lambda):=\int e^{i\langle\lambda, x\rangle} h_{n}(x) d x=\int e^{-n k(a, \lambda / \sqrt{n})} d \mu_{A, n}(a),
$$

where

$$
k(a, \lambda):=i\langle a, \lambda\rangle+\ln \phi\left(m^{\mathrm{inv}}(a)\right)-\ln \phi\left(m^{\mathrm{inv}}(a)+i \lambda\right) .
$$

P r o of. We have

$$
\begin{aligned}
& \widehat{h}_{n}(\lambda)=\int e^{i\langle\lambda, x / \sqrt{n}\rangle} g_{n}(x) d x
\end{aligned}
$$

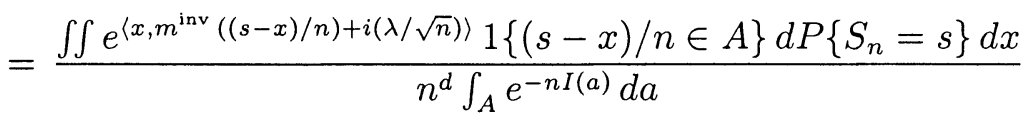

$$
\begin{aligned}
& =\frac{\iint e^{\left\langle s-n a, m^{\mathrm{inv}}(a)+i(\lambda / \sqrt{n})\right\rangle} 1\{a \in A\} d P\left\{S_{n}=s\right\} d a}{\int_{A} e^{-n I(a)} d a} \\
& =\frac{\int_{A} e^{-n\left\langle a, m^{\mathrm{inv}}(a)+i(\lambda / \sqrt{n})\right\rangle}\left(\phi\left(m^{\mathrm{inv}}(a)+i(\lambda / \sqrt{n})\right)\right)^{n} d a}{\int_{A} e^{-n I(a)} d a} \\
& =\frac{\int_{A} e^{-n I(a)} e^{-n k(a, \lambda / \sqrt{n})} d a}{\int_{A} e^{-n I(a)} d a}=\int e^{-n k(a, \lambda / \sqrt{n})} d \mu_{A, n}(a),
\end{aligned}
$$

where we used again (3.1). Lemma 3.4 is proved.

We are now in position to complete the proof of ii) of Theorem 2.1' when $A$ is relatively compact. Let $\psi$ and $\psi_{n}$ be the densities with characteristic functions

$$
\begin{aligned}
\widehat{\psi}_{n}(\lambda) & :=\int_{A} \exp \left(-\frac{1}{2}\langle V(a) \lambda, \lambda\rangle\right) d \mu_{A, n}(a) \\
\widehat{\psi}(\lambda) & :=\int_{\Lambda_{I(A)}} \exp \left(-\frac{1}{2}\langle V(a) \lambda, \lambda\rangle\right) d \mu_{A}(a)
\end{aligned}
$$

One sees that $\psi_{n}$ and $\psi$ are just mixtures of normal densities with respect to the mixing measures $\mu_{A, n}$ and $\mu_{A}$, and in particular,

$$
\psi(x)=\int_{\Lambda_{I(A)}}(2 \pi)^{-d / 2}|V(a)|^{-1 / 2} \exp \left(-\frac{1}{2}\left\langle V(a)^{-1} x, x\right\rangle\right) d \mu_{A}(a) .
$$

Using the Fourier inversion formula gives

$$
\begin{aligned}
\left|h_{n}(0)-\psi_{n}(0)\right| & \leqslant \int\left|\widehat{h}_{n}(\lambda)-\widehat{\psi}_{n}(\lambda)\right| d \lambda \\
& \leqslant \int_{A} \int\left|e^{-n k(a, \lambda / \sqrt{n})}-e^{-1 / 2\langle V(a) \lambda, \lambda\rangle}\right| d \lambda d \mu_{A, n}(a) .
\end{aligned}
$$


Our proof now follows closely that of the local central limit theorem as in [28, Theorem XV.5.2].

Since $\operatorname{cl}(A)$ is compact and $V(a)=($ Hess $\ln \phi)\left(m^{\text {inv }}(a)\right)$ is the variance function,

$$
\lim _{\varepsilon \rightarrow 0} \limsup \sup _{n \rightarrow \infty} \sup _{a \in A}|\lambda| \leqslant \varepsilon n^{1 / 6}\left|n k\left(a, \frac{\lambda}{\sqrt{n}}\right)-\frac{1}{2}\langle V(a) \lambda, \lambda\rangle\right|=0 .
$$

Hence, for any $C>0$,

$$
\lim _{n \rightarrow \infty} \int_{A} \int_{|\lambda| \leqslant C}\left|e^{-n k(a, \lambda / \sqrt{n})}-e^{-\langle V(a) \lambda, \lambda\rangle / 2}\right| d \lambda d \mu_{A, n}(a)=0 .
$$

Moreover, (2.3) and the relative compactness of $A$ yield for any $n$ large enough, any $a \in A$ and any $\varepsilon$ small enough, in the range $|\lambda| \leqslant \varepsilon \sqrt{n}$,

$$
\operatorname{Re}\left(n k\left(a, \frac{\lambda}{\sqrt{n}}\right)\right) \geqslant \frac{1}{4}\langle V(a) \lambda, \lambda\rangle \geqslant \frac{v(a)}{4}|\lambda|^{2},
$$

where $v(a)$ is the smallest eigenvalue of $V(a)$. Therefore, for any $C>0$,

$$
\begin{gathered}
\int_{A} \int_{C \leqslant|\lambda| \leqslant \varepsilon n^{1 / 2}}\left|e^{-n k(a, \lambda / \sqrt{n})}\right| d \lambda d \mu_{A, n}(a) \\
\leqslant \int_{A} \int_{C \leqslant|\lambda|} e^{-v(a)|\lambda|^{2} / 4} d \lambda d \mu_{A, n}(a)
\end{gathered}
$$

tends to 0 as $n \rightarrow \infty$ and $C \rightarrow \infty$.

Furthermore, we have

$$
\begin{aligned}
& \int_{A} \int_{|\lambda|>C} \exp \left(-\frac{1}{2}\langle V(a) \lambda, \lambda\rangle\right) d \lambda d \mu_{A, n}(a) \\
& \quad \leqslant \int_{A} \int_{|\lambda|^{2} \geqslant C^{2}} \exp \left(-\frac{1}{2} v(a)|\lambda|^{2}\right) d \lambda d \mu_{A, n}(a) .
\end{aligned}
$$

Now, since we assume that $\operatorname{cl} A$ is compact, since $v(\cdot)$ is continuous on $\mathscr{N}$, and since $\mu_{A, n}$ has total mass $1,(3.4)$ tends to 0 as $n \rightarrow \infty$ and then $C \rightarrow \infty$.

Finally,

$$
\begin{aligned}
& \int_{A} \int_{|\lambda| \geqslant \varepsilon \sqrt{n}} e^{-n k(a, \lambda / \sqrt{n})} d \lambda d \mu_{A, n}(a) \\
& \quad \leqslant \int_{A} \int_{|u| \geqslant \varepsilon}\left|\frac{\phi\left(m^{\text {inv }}(a)+i u\right)}{\phi\left(m^{\text {inv }}(a)\right)}\right|^{n} d u d \mu_{A, n}(a) n^{d / 2} \\
& \quad \leqslant \sup _{a \in A|v| \geqslant \varepsilon}\left|\frac{\phi\left(m^{\text {inv }}(a)+i v\right)}{\phi\left(m^{\text {inv }}(a)\right)}\right|^{n-r} n^{d / 2} \int_{A} \int_{|u| \geqslant \varepsilon}\left|\widehat{p}_{a}(u)\right|^{r} d u d \mu_{A, n}(a) .
\end{aligned}
$$


The map $a \mapsto \sup _{|v| \geqslant \varepsilon} \phi\left(m^{\text {inv }}(a)+i v\right) / \phi\left(m^{\text {inv }}(a)\right)$ is continuous over the compact $\operatorname{cl} A$ and less than 1. Thus,

$$
\lim _{n \rightarrow \infty} \sup _{a \in \operatorname{cl}} \sup _{A|v| \geqslant \varepsilon} n^{d / 2}\left|\frac{\phi\left(m^{\text {inv }}(a+i v)\right)}{\phi\left(m^{\text {inv }}(a)\right)}\right|^{n-r}=0 .
$$

Since $\mu_{A, n}$ is concentrated on the compact cl $A$ and (2.2) holds, we deduce that for any $\varepsilon>0$,

$$
\lim _{n \rightarrow \infty} \int_{A} \int_{|\lambda| \geqslant \varepsilon \sqrt{n}}\left|e^{-n k(a, \lambda / n)}\right| d \lambda d \mu_{A, n}(a)=0 .
$$

Combine (3.2)-(3.5) to obtain that

$$
\lim _{n \rightarrow \infty}\left|h_{n}(0)-\psi_{n}(0)\right|=0
$$

Now, notice that the function

$$
a \longmapsto(2 \pi)^{-d / 2}|V(a)|^{-1 / 2} \exp \left(-\frac{1}{2}\left\langle V(a)^{-1} x, x\right\rangle\right)
$$

is continuous and bounded. Thus, along a subsequence $n_{k}$ for which $\mu_{A, n_{k}}$ converges weakly* to $\mu_{A}$, we obtain

$$
\lim _{k \rightarrow \infty} \psi_{n_{k}}(0)=\psi(0)=\int(2 \pi)^{-d / 2}|V(a)|^{-1 / 2} d \mu_{A}(a) .
$$

Combine (3.6) and (3.7) to get the statement ii) of Theorem $2.1^{\prime}$ for relatively compact sets $A$.

In order to extend the result to arbitrary sets $A$, we first prove Corcllary 2.1 for relatively compact sets.

$\mathrm{P}$ r o o f of $\mathrm{C}$ or o ll a r y 2.1 . Assume that $A$ is relatively compact. Set $c=I(A)=\operatorname{essinf}\{I(a): a \in A\}$. Then for any $\varepsilon>0$,

$$
\int_{A} e^{-n I(a)} d a \geqslant \int_{A \cap \Gamma_{c+\varepsilon}} e^{-n I(a)} d a \geqslant e^{-n(c+\varepsilon)}\left|A \cap \Gamma_{c+\varepsilon}\right| .
$$

Thus, using Theorem 2.1-ii) and Comment 2.2 yields

$$
\liminf _{n \rightarrow \infty} n^{-1} \ln P\left\{S_{n} \in n A\right\} \geqslant-c .
$$

Furthermore,

$$
\begin{aligned}
\int_{A} e^{-n I(a)} d a & \leqslant \int_{\Gamma_{c}^{c}} e^{-n I(a)} d a=e^{-n c} \int_{\Gamma_{c}^{c}} e^{-n(I(a)-c)} d a \\
& \leqslant e^{-n c} \int_{\Gamma_{c}^{c}} e^{-(I(a)-c)} d a \leqslant e^{-n c} e^{c} \int e^{-I(x)} d x
\end{aligned}
$$


and therefore,

$$
\limsup _{n \rightarrow \infty} n^{-1} \ln P\left\{S_{n} \in n A\right\} \leqslant-c .
$$

Thus Corollary 2.1 holds for relatively compact $A$. The same proof shows that it holds for arbitrary set $A$ provided Theorem $2.1^{\prime}$-ii) holds.

We now conclude the proof of Theorems 2.1 and $2.1^{\prime}$. If $A$ is not relatively compact and $c=I(A)<\infty$, write $A=\left(A \cap \Gamma_{c+2}\right) \cup\left(A \cap \Gamma_{c+2}^{c}\right)$. We evaluate $P\left\{S_{n} \in n\left(A \cap \Gamma_{c+2}\right)\right\}$ with the help of Theorem 2.1'-ii) (in the relatively compact case). And then the rough large deviation estimate (1.2) shows that for $n$ large enough

$$
P\left\{S_{n} \in n\left(A \cap \Gamma_{c+2}\right)\right\} \leqslant P\left\{S_{n} \in n \Gamma_{c+2}\right\} \leqslant e^{-n(c+1)},
$$

which is of smaller order than $P\left\{S_{n} \in n\left(A \cap \Gamma_{c+2}\right)\right\}$ thanks to Corollary 2.1 (applied to the relatively compact set $A \cap \Gamma_{c+2}$ ).

To prove Theorem 2.1, notice that (3.6) yields

$$
P\left\{S_{n} \in n A\right\}=\left(\frac{n}{2 \pi}\right)^{d / 2} \int_{A}|V(a)|^{-1 / 2} e^{-n I(a)} d a+o\left(n^{d / 2} \int_{A} e^{-n I(a)} d a\right) .
$$

But using (2.3), the notation introduced in Comment 2.2, and the relative compactness of $A$,

$$
\begin{aligned}
0 & \leqslant v_{U}(\operatorname{cl} A)^{-1 / 2} \int_{A} e^{-n I(a)} d a \leqslant \int_{A}|V(a)|^{-1 / 2} e^{-n I(a)} d a \\
& \leqslant v_{L}(\operatorname{cl} A)^{-1 / 2} \int_{A} e^{-n I(a)} d a<\infty .
\end{aligned}
$$

Therefore, (3.8) implies Theorem 2.1. Notice that the relative compactness of $A$ is mainly used to ensure that the crude bounds we use in (3.9) are not 0 or infinite.

\section{Proofs of the consequences of Theorem 2.1.}

$\mathrm{Pr}$ o of of $\mathrm{Cor}$ oll a r y 2.2. This is only a restatement of Karamata's Tauberian theorem which can be found, e.g., in [7].

$\mathrm{Pro}$ of of Corollary 2.3. This is just a restatement of de Bruijn's [18] exponential Tauberian theorem; see, e.g., [7].

$\mathrm{Pr}$ o of of $\mathrm{Proposition} 2.1$. Our goal is to estimate integrals of the form $\int_{A} f(a) e^{-n I(a)} d a$ for any bounded $C^{\infty}$ function $f$ on $\mathbf{R}^{d}$. Taking $f(a)=|V(a)|^{-1 / 2}$ will lead the result. The use of an arbitrary $C^{\infty}$ function will allow us to calculate the measure $\mu_{A}$. Notice that up to changing $f$ into $f+\|f\|_{\infty}$, we can assume that $f$ is nonnegative.

Let us denote $c=I(A)$ and recall that $\mathscr{D}$ is the extension of $D=A \cap \Lambda_{c}$ on $\Lambda_{c}$ if $D$ has a boundary, and $\mathscr{D}=D$ otherwise. In what follows we 
shall assume that $\mathscr{D}$ can be covered by one chart. It may happen that we need an atlas, but the extension is trivial and left to the reader. Hence, let $m\left(t\left(u_{1}, \ldots, u_{k}\right)\right),\left(u_{1}, \ldots, u_{k}\right) \in U \in \mathbf{R}^{k}$, be a parametrization of $\mathscr{D}$. Let $T \mathscr{D}$ and $N \mathscr{D}$ be the tangent and normal bundles of $\mathscr{D}$ immersed in $\mathbf{R}^{d}$. Let $\omega^{1}(p), \ldots, \omega^{d-k}(p), p \in \mathscr{D}$, be an orthonormal moving frame in $N \mathscr{D}$ such that $\omega^{d-k}(p)$ is normal at $p$ to $\Lambda_{c} \supset \mathscr{D}$ when $p \in D$. It is convenient to choose $\omega^{i}(p), 1 \leqslant i \leqslant d-k-1$, to be the principal directions of the submanifold $\mathscr{S}_{p} \partial A$, so that they diagonalize its second fundamental form at $p$. Let $\Pi_{i}(p)$, $1 \leqslant i \leqslant d-k$, be the second fundamental forms of the manifold $\mathscr{D}$ (immersed in $\mathbf{R}^{d}$ ) at $p \in \mathscr{D}$ along the normal vectors $\omega^{i}(p)$. We denote by

$$
G_{\alpha, \beta}^{j}(p):=\Pi_{j}(p)\left(\frac{\partial m \circ t}{\partial u_{\alpha}}, \frac{\partial m \circ t}{\partial u_{\beta}}\right), \quad 1 \leqslant \alpha, \quad \beta \leqslant d-k, \quad p \in \mathscr{D} .
$$

In all this section, we use Einstein's summation convention, writing, e.g., $h_{i} \omega^{i}(p)$ for $\sum_{1 \leqslant i \leqslant d-k} h_{i} \omega^{i}(p)$. For small $\eta$ consider the tubular neighborhood $\mathscr{D}(\eta)$ of $\mathscr{D}$ defined by

$$
\mathscr{D}(\eta):=\left\{p+h_{i} \omega^{i}(p): p \in \mathscr{D},|h| \leqslant \eta\right\} .
$$

Let $\widehat{h}:=\left(h_{1}, \ldots, h_{d-k-1}\right)$. If $S$ is a matrix, we denote by $\|\mid S\| \|:=$ $\sup \{|S x|:|x| \leqslant 1\}$ its norm. We shall need the following fact. If $S$ is a symmetric matrix, then for $|h| \leqslant \eta$ and $h_{d-k} \geqslant 0$,

$$
\begin{aligned}
|\langle S h, h\rangle-\langle S \widehat{h}, \widehat{h}\rangle| & =\left|2\left\langle S h, \omega^{d-k}(p)\right\rangle h_{d-k}+\left\langle S \omega^{d-k}(p), \omega^{d-k}(p)\right\rangle h_{d-k}^{2}\right| \\
& \leqslant 3 \eta\||| S \mid\| h_{d-k} .
\end{aligned}
$$

Now, since $A \cap \Lambda_{c}=D \subset \mathscr{D}$ and $\partial A$ is smooth and $\omega_{d-k}$ is an outward normal to $\Gamma_{c}$, there exists a function $g(p, \widehat{h})$ such that

$$
A \cap \mathscr{D}(\eta)=\left\{p+h_{i} \omega^{i}(p): p \in \mathscr{D}, h_{d-k} \geqslant g(p, \widehat{h}),|h| \leqslant \eta\right\} .
$$

In all what follows, we denote by $C$ a constant which depends on the distribution of $X$, on the set $A$, but which does not depend on $\eta$ or $\varepsilon$ to be introduced later, provided $\eta$ is small enough. The constant may vary from place to place, although we keep the same letter. In order to develop a Laplace type approximation, we need first to obtain some bounds on the functions playing some role in the integral $\int_{A} f(a) e^{-n I(a)} d a$.

Since $\partial A$ and $\Lambda_{c}$ are tangent along $D$, the differential $D_{\hat{h}} g(p, 0)$ of $\widehat{h} \mapsto g(p, \widehat{h})$ is 0 for $p \in D$. Let $W(p)$ be the Hessian of $\widehat{h} \mapsto g(p, \widehat{h})$ at $\widehat{h}=0$. The quadratic form induced by $W(p)$ coincide with the second fundamental form of $\mathscr{S}_{p} \partial A$ on $T_{p}\left(\mathscr{S}_{p} \partial A\right)$. Write $W(p)^{i j}=\left\langle W(p) \omega^{i}, \omega^{j}\right\rangle$. Our choice of $\omega^{i}(p)$ yields

$$
g(p, \widehat{h})=\frac{1}{2}\langle W(p) \widehat{h}, \widehat{h}\rangle+O\left(|\widehat{h}|^{3}\right)=\widehat{h}_{i}^{2} W(p)^{i i}+O\left(|\widehat{h}|^{3}\right)
$$




$$
\left\{\begin{array}{l}
\leqslant \frac{1}{2} \widehat{h}_{i}^{2}\left(W(p)^{i i}+C \eta\right) \\
\geqslant \frac{1}{2} \widehat{h}_{i}^{2}\left(W(p)^{i i}-C \eta\right)
\end{array} .\right.
$$

Hence, for $p \in D$, we can sandwich $\mathscr{S}_{p} \partial A$ between two paraboloids,

$$
\frac{1}{2}\langle(W(p)-C \eta \operatorname{Id}) \widehat{h}, \widehat{h}\rangle \leqslant g(p, \widehat{h}) \leqslant \frac{1}{2}\langle(W(p)+C \eta \operatorname{Id}) \widehat{h}, \widehat{h}\rangle .
$$

Furthermore, if $p \in \mathscr{D} / D$, we have $g(p, 0)>0$ and

$$
g(p, 0)-C|\widehat{h}| \leqslant g(p, \widehat{h}) \leqslant g(p, 0)+C|\widehat{h}| .
$$

We expand $I(\cdot)$, using the fact that $D m^{\text {inv }}(p)=V(p)^{-1}$, to obtain

$$
\begin{aligned}
I(p+q) & =\left\langle m^{\mathrm{inv}}(p+q), p+q\right\rangle-\ln \phi\left(m^{\mathrm{inv}}(p+q)\right) \\
& =I(p)+\left\langle m^{\mathrm{inv}}(p), q\right\rangle+\frac{1}{2}\left\langle V(p)^{-1} q, q\right\rangle+O\left(|q|^{3}\right) .
\end{aligned}
$$

Notice that $m^{\text {inv }}(p)$ is normal to $\Lambda_{c}$ at $p$, and thus colinear to $\omega^{d-k}(p)$. Also $\inf \left\{\left|m^{\text {inv }}(p)\right|: p \in \Lambda_{c}\right\}>0$ (for $c>0$ ) and (2.3) holds. Thus, use of (4.1) yields

$$
\begin{aligned}
& I\left(p+h_{i} \omega^{i}(p)\right)=c+\left|m^{\mathrm{inv}}(p)\right| h_{d-k}+\frac{1}{2}\left\langle V(p)^{-1} h, h\right\rangle+O\left(|h|^{3}\right) \\
& \left\{\begin{array}{l}
\leqslant c+\left(\left|m^{\mathrm{inv}}(p)\right|+\frac{1+2 \eta}{2} 3 \eta||\left|V(p)^{-1}\right|||\right) h_{d-k}+\frac{1+C \eta}{2}\left\langle V(p)^{-1} \widehat{h}, \widehat{h}\right\rangle \\
\geqslant c+\left(\left|m^{\mathrm{inv}}(p)\right|-\frac{1+2 \eta}{2} 3 \eta||\left|V(p)^{-1}\right|||\right) h_{d-k}+\frac{1-C \eta}{2}\left\langle V(p)^{-1} \widehat{h}, \widehat{h}\right\rangle
\end{array}\right.
\end{aligned}
$$

Following Weyl [46] in doing hardly «more than what could have been accomplished by any student in a course of calculus» in 1939, we have for any $x \in \mathbf{R}^{d}$,

$$
d x=\operatorname{det}\left(\delta_{\alpha, \beta}+h_{j} G_{\alpha, \beta}^{j}(p)\right)_{1 \leqslant \alpha, \beta \leqslant d-k} d h_{d-k} d \widehat{h} d \mathscr{M}(p),
$$

where $d \widehat{h}=d h_{1} \cdots d h_{d-k-1}$ and $\delta_{\alpha, \beta}$ is the Kronecker symbol (i.e., equal to 1 if $\alpha=\beta$ and 0 otherwise). But for $h \leqslant \eta$, we have

$$
1-C \eta \leqslant \operatorname{det}\left(\delta_{\alpha, \beta}+h_{j} G_{\alpha, \beta}^{j}(p)\right)_{q \leqslant \alpha, \beta \leqslant d-k} \leqslant 1+C \eta .
$$

We set $v_{d-k}:=n h_{d-k}, \quad v_{i}:=\sqrt{n} h_{i}, 1 \leqslant i \leqslant d-k-1$, and $\widehat{v}:=$ $\sum_{1 \leqslant i \leqslant d-k-1} v_{i} \omega^{i}(p)$ and $d \widehat{v}=d v_{1} \cdots d v_{d-k-1}$.

We have now all the bounds in hand to obtain a Laplace type approximation of the leading part of our integral. 
Since $A \cap \Gamma_{c}=D$, for $\varepsilon$ small enough, $A \cap \Gamma_{c+\varepsilon}$ is a subset of the tubular neighborhood $\mathscr{D}(\eta)$. Thus, putting all the pieces together, we have for any $\varepsilon$ small enough,

$$
\begin{aligned}
\int_{A \cap \Gamma_{c+\varepsilon}} f(a) e^{-n I(a)} d a & \\
=\int_{\mathscr{D}} \int_{|\widehat{h}| \leqslant C \sqrt{\varepsilon}} \int_{0 \leqslant h_{d-k} \leqslant C \varepsilon} & f\left(p+h_{i} \omega^{i}(p)\right) e^{-n I\left(p+h_{i} \omega^{i}(p)\right)} \\
& \times 1\left\{I\left(p+h_{i} \omega^{i}(p)\right) \leqslant c+\varepsilon, h_{d-k} \geqslant g(p, \widehat{h})\right\} \\
& \times \operatorname{det}\left(\delta_{\alpha, \beta}+h_{j} G_{\alpha, \beta}^{j}(p)\right) d h_{d-k} d \widehat{h} d \mathscr{M}(p) \leqslant(I)+(I I),
\end{aligned}
$$

where (here we combine all the previous bounds and use also the differentiability of $f$ )

$$
\begin{aligned}
(I):=\int_{D} \int_{\widehat{v} \mid \leqslant C \sqrt{\varepsilon n}} \int_{0 \leqslant v_{d-k} \leqslant C \varepsilon n}(f(p)+C \eta) e^{-n c} \\
\quad \times \exp \left\{-\left(\left|m^{\text {inv }}(p)\right|-C \eta\right) v_{d-k}\right\} \exp \left\{-\frac{1-C \eta}{2}\left\langle V(p)^{-1} \widehat{v}, \widehat{v}\right\rangle\right\} \\
\quad \times 1\left\{v_{d-k} \geqslant \frac{1}{2}\langle(W(p)-C \eta \mathrm{Id}) \widehat{v}, \widehat{v}\rangle\right\} \\
\quad \times(1+C \eta) d v_{d-k} d \widehat{v} d \mathscr{M}(p) n^{-(d-k+1) / 2}
\end{aligned}
$$

and

$$
\begin{aligned}
(I I):=\int_{\mathscr{D} / D} & \|f\|_{\infty} \int_{|\widehat{v}| \leqslant C \sqrt{\varepsilon n}} \int_{0 \leqslant v_{d-k} \leqslant C \varepsilon n} e^{-n c} \exp \left\{-\left(\left|m^{\mathrm{inv}}(p)\right|-\eta C\right) v_{d-k}\right\} \\
& \times \exp \left\{-\frac{1-C \eta}{2}\left\langle V(p)^{-1} \widehat{v}, \widehat{v}\right\rangle\right\} 1\left\{v_{d-k} \geqslant n g(p, 0)-\sqrt{n} C\|\widehat{v}\|\right\} \\
& \times(1+C \eta) d v_{d-k} d \widehat{v} d \mathscr{M}(p) n^{-(d-k+1) / 2} .
\end{aligned}
$$

We can now bound $(I)$ and $(I I)$. For $(I)$ we have

$$
\begin{aligned}
& (I) \leqslant \int_{D} \int_{\mathbf{R}^{d-k-1}}(f(p)+C \eta) e^{-n c} \\
& \times \frac{\exp \left\{-\left(\left|m^{\text {inv }}(p)\right|-\eta C\right) \frac{1}{2}\langle(W(p)-C \eta \operatorname{Id}) \widehat{v}, \widehat{v}\rangle\right\}}{\left|m^{\text {inv }}(p)\right|-C \eta} \\
& \times \exp \left\{-\frac{1-C \eta}{2}\left\langle V(p)^{-1} \widehat{v}, \widehat{v}\right\rangle\right\}\left(1+C r_{i}\right) d \widehat{v} d \mathscr{M}(p) n^{-(d-k+1) / 2} \\
& \leqslant e^{-n c} n^{-(d-k+1) / 2} \int_{D} \int_{\mathbf{R}^{d-k-1}}(f(p)+C \eta)\left(\left|m^{\mathrm{inv}}(p)\right|-C \eta\right)^{-1} \\
& \times \exp \left(-\frac{1}{2}\left\langle\left(\left(\left|m^{\mathrm{inv}}(p)\right|-\eta C\right)(W(p)-C \eta \mathrm{Id})\right.\right.\right. \\
& \left.\left.\left.+(1-C \eta) V(p)^{-1}\right) \widehat{v}, \widehat{v}\right\rangle\right)(1+C \eta) d \widehat{v} d \mathscr{M}(p) .
\end{aligned}
$$


Recalling that $W(p)$ and $-V(p)^{-1}\left|m^{\mathrm{inv}}(p)\right|^{-1}$ restricted in the tangent space $\widetilde{N}_{p}(D)$ of $\mathscr{S}_{p} \Lambda_{c}$ and $\mathscr{S} \partial A$ coincide with the fundamental forms of $\mathscr{S}_{p} \Lambda_{c}$ and $\mathscr{S}_{p} \partial A$, we obtain

$$
\begin{aligned}
(I) \leqslant & e^{-n c} n^{-(d-k+1) / 2} \\
& \times \int_{D}(f(p)+C \eta)(2 \pi)^{(d-k-1) / 2}\left(\left|m^{\mathrm{inv}}(p)\right|-C \eta\right)^{-1}\left|m^{\mathrm{inv}}(p)\right|^{-(d-k-1) / 2} \\
& \times\left|\operatorname{det}\left(\left(1-C \eta\left|m^{\mathrm{inv}}(p)\right|^{-1}\right)\left(\mathscr{W}_{\mathscr{S}_{p} \Lambda_{c}, p}-C \eta \mathrm{Id}\right)-(1-C \eta) \mathscr{W}_{\mathscr{S}_{p} A, p}\right)\right|^{-1 / 2} \\
& \times(1+C \eta) d \mathscr{M}(p) .
\end{aligned}
$$

As far as $(I I)$ is concerned, it can be neglected since

$$
\begin{aligned}
& e^{n c} n^{(d-k+1) / 2}(I I) \\
& \leqslant \int_{\mathscr{D} / D}\|f\|_{\infty} \int_{\mathbf{R}^{d-k-1}} \exp \left\{-\left(\left|m^{\mathrm{inv}}(p)\right|-\eta C\right\}(n g(p, 0)-\sqrt{n}\|\widehat{v}\|)_{+}\right. \\
& \quad \times \exp \left\{-\frac{1-C \eta}{2}\left\langle V(p)^{-1} \widehat{v}, \widehat{v}\right\rangle\right\}(1+C \eta) d \widehat{v} d \mathscr{M}(p) .
\end{aligned}
$$

For any fixed $p \in \mathscr{D} / D$ the function

$$
(p, \widehat{v}) \longmapsto \exp \left\{-\left(\left|m^{\text {inv }}(p)\right|-\eta C\right)(n g(p, 0)-\sqrt{n}\|\widehat{v}\|)_{+}\right\}
$$

converges to 0 almost everywhere on $(\mathscr{D} / D) \times \mathbf{R}^{d-k-1}$ and is less than 1 . Thus Lebesgue's dominated convergence theorem yields

$$
\lim _{n \rightarrow \infty} e^{n c} n^{(d-k+1) / 2}(I I)=0 .
$$

From what preceds, we deduce that for $\varepsilon$ small enough,

$$
\begin{aligned}
& \limsup _{n \rightarrow \infty} e^{n c} n^{(d-k+1) / 2} \int_{A \cap \Gamma_{c+\varepsilon}} f(a) e^{-n I(a)} d a \\
& \leqslant \int_{D} f(p)(2 \pi)^{(d-k-1) / 2}\left|m^{\text {inv }}(p)\right|^{-(d-k+1) / 2} \mid \\
& \quad \times\left.\operatorname{det}\left(\mathscr{W}_{\mathscr{S}_{c}, p}-\mathscr{W}_{\mathscr{S} \partial A, p}\right)\right|^{-1 / 2} d \mathscr{M}(p)+C \eta .
\end{aligned}
$$

For the lower bound, a very similar calculation (but somewhat easier since we can restrict the integration over $D$ instead of $\mathscr{D}$ ) yields for any $\varepsilon$ small enough,

$$
\begin{aligned}
\liminf _{n \rightarrow \infty} e^{n c} n^{(d-k+1) / 2} \int_{A \cap \Gamma_{c+\varepsilon}} f(a) e^{-n I(a)} d a \\
\geqslant \int_{D} f(p)(2 \pi)^{(d-k-1) / 2}\left|m^{\text {inv }}(p)\right|^{(d-k-1) / 2} \\
\quad \times \operatorname{det}\left(\mathscr{W}_{\mathscr{S}_{p} \Lambda_{c}, p}-\mathscr{W}_{\mathscr{S}_{p} \partial A, p}\right)^{-1 / 2} d \mathscr{M}(p) .
\end{aligned}
$$


Furthermore,

$$
\int_{A \cap \Gamma_{c+\varepsilon}^{c}} f(a) e^{-n I(a)} d a \leqslant\|f\|_{\infty} \int_{\Gamma_{c+\varepsilon}^{c}} e^{-n I(a)} d a \leqslant C n^{d} e^{-n(c+\varepsilon)} .
$$

Therefore, since $\eta$ can be arbitrary small,

$$
\begin{aligned}
& \lim _{n \rightarrow \infty} e^{n c} n^{(d-k+1) / 2} \int_{A} f(a) e^{-n I(a)} d a \\
& =\int_{D} f(p)(2 \pi)^{(d-k-1) / 2}\left|m^{\text {inv }}(p)\right|^{(d-k-1) / 2} \\
& \quad \times \operatorname{det}\left(\mathscr{W}_{\mathscr{S}_{p} A(p), p}-\mathscr{W}_{\mathscr{S}_{p} \Lambda_{c}, p}\right)^{-1 / 2} d \mathscr{M}(p),
\end{aligned}
$$

and this completes the proof of Proposition 2.1.

P r o of of $\mathrm{C}$ or o 11 a r y 2.4. Let $\mathscr{M}$ be the Riemannian measure on $\Lambda_{c}$. Let $A=\Gamma_{c}$ and let $B$ be a submanifold of $\Lambda_{c}$ with $\mathscr{M}(B)>0$. Let $\widetilde{B}$ be the truncated cone with base $B$ defined by

$$
\widetilde{B}:=\left\{s x \in \Gamma_{c}^{c}: x \in B, s \geqslant 0\right\} .
$$

Theorem 2.1 and Proposition 2.1 apply with $k=d-1$ to give

$$
\begin{aligned}
P\left\{S_{n} \in n \widetilde{B} \mid S_{n} \in n A\right\} & =\frac{P\left\{S_{n} \in n \widetilde{B}\right\}}{P\left\{S_{n} \in n A\right\}} \\
& \sim \frac{\int_{B}\left[|V(p)|^{1 / 2}\left|m^{\text {inv }}(p)\right|\right]^{-1} d \mathscr{M}(p)}{\int_{\Lambda_{c}}|V(p)|^{-1 / 2}\left|m^{\text {inv }}(p)\right| d \mathscr{M}(p)} \quad \text { as } \quad n \rightarrow \infty .
\end{aligned}
$$

5. Proof of Theorem 2.2 and Corollary 2.5. The proofs are very similar to those of Theorem 2.1 and Corollary 2.1.

Changing $X_{i}=\left(X_{i, 1}, \ldots, X_{i, d}\right)$ to $X_{i} / h=\left(X_{i, 1} / h_{1}, \ldots, X_{i, d} / h_{d}\right)$ and $A$ to $A / h$, we can assume that $h=(1, \ldots, 1)$. The symbol $\sum_{k}$ means $\sum_{k \in \mathbf{Z}^{d}}$ in this section.

The analogue of Lemma 3.1 is the following.

Lemma 5.1. For any $\eta>0$ and any $\alpha>0$,

$$
\sum_{k} 1\left\{I\left(\frac{k}{n}\right) \geqslant \alpha\right\} e^{-n \eta I(k / n)}=O\left(n^{d} e^{-n \eta \alpha}\right)
$$

as $n \rightarrow \infty$.

Pr o o f. Since $I$ is convex, for any $l \in \mathbf{N}$,

$$
\sharp\left\{k: I\left(\frac{k}{n}\right) \in[\alpha+l, \alpha+l+1]\right\} \leqslant \sharp\left\{k: I\left(\frac{k}{n}\right) \in[0,1]\right\}=O\left(n^{d}\right)
$$

as $n \rightarrow \infty$. Consequently,

$$
\begin{aligned}
\sum_{k} 1\left\{I\left(\frac{k}{n}\right) \geqslant \alpha\right\} e^{-n \eta I(k / n)} & \leqslant \sum_{l \in \mathrm{N}} \sharp\left\{k: \alpha+l \leqslant I\left(\frac{k}{n}\right) \leqslant \alpha+l+1\right\} e^{-n \eta(\alpha+l)} \\
& \leqslant O\left(n^{d}\right) e^{-n \eta \alpha} \sum_{k} e^{-n \eta l} .
\end{aligned}
$$


The analogue of Lemma 3.2 is slightly more complicated since $I(A)$ is now defined differently.

Lemma 5.2. Let $c_{n}:=\inf \{I(k / n): k / n \in A\}$. Let $n_{k}$ be a subsequence such that $\lim _{k \rightarrow \infty} c_{n_{k}}=: c \in(0, \infty)$ exists. Then the sequence $\mu_{A, n_{k}}$ is tight, and any limiting measure is concentrated on $\Lambda_{c}$.

$\mathrm{P}$ r o o f. For simplicity, we write $n$ for $n_{k}$. Let $\alpha>c$ and $\varepsilon>0$ be such that $c_{n}+2 \varepsilon<\alpha$ for any $n$ large enough. Let $b_{n}:=\left(c_{n}+\alpha\right) / 2$ and $\eta_{n}:=1-\left(b_{n} / \alpha\right)$. If $I(a) \geqslant \alpha$, then $I(a)-b_{n} \geqslant \eta_{n} I(a)$. Hence, for $n$ large enough,

$$
\begin{aligned}
\mu_{A, n}\left(\Gamma_{\alpha}^{c}\right) & \leqslant e^{-n b_{n}} \frac{\sum_{k} 1\{I(k / n) \geqslant \alpha\} e^{-n \eta_{n} I(k / n)}}{e^{-n\left(c_{n}+\varepsilon\right)} \sharp\left\{k: k / n \in A \cap \Gamma_{c_{n}+\varepsilon}\right\}} \\
& \leqslant e^{-n\left(b_{n}-c_{n}-\varepsilon\right)} \sum_{k} 1\left\{I\left(\frac{k}{n}\right) \geqslant \alpha\right\} e^{-n \eta_{n} I(k / n)} .
\end{aligned}
$$

Notice that $\eta:=\lim _{n \rightarrow \infty} \eta_{n}=(\alpha-c) / 2 \alpha>0$ and that $\lim _{n \rightarrow \infty}\left(b_{n}-c_{n}-\varepsilon\right)=$ $(\alpha-c-2 \varepsilon) / 2>0$. Hence, for $n$ large enough,

$$
\mu_{A, n}\left(\Gamma_{\alpha}^{c}\right) \leqslant e^{-n(\alpha-c-2 \varepsilon) / 4} \sum_{k} 1\left\{I\left(\frac{k}{n}\right) \geqslant \alpha\right\} e^{-n \eta I(k / n) / 2}=o(1)
$$

thanks to Lemma 5.1.

The proof now follows closely that of Theorem 2.1 and we shall omit some details.

Lemma 5.3. The following equality holds true:

$$
\begin{gathered}
\sum_{l} \sum_{s} e^{\left\langle m^{\mathrm{inv}}((s-l) / n), l\right\rangle} 1\left\{\frac{s-l}{n} \in A\right\} P\left\{S_{n}=s\right\}=\sum_{k} 1\left\{\frac{k}{n} \in A\right\} e^{-n I(k / n)} . \\
\text { P r o o f. Let } k=s-l \text { and notice that } \\
\sum_{k} \sum_{s} e^{\left\langle m^{\text {inv }}(k / n), s-k\right\rangle} 1\left\{\frac{k}{n} \in A\right\} P\left\{S_{n}=s\right\} \\
=\sum_{k} 1\left\{\frac{k}{n} \in A\right\} e^{-n\left\langle m^{\text {inv }}(k / n), k / n\right\rangle} \phi\left(m^{\text {inv }}\left(\frac{k}{n}\right)\right)^{n} .
\end{gathered}
$$

On $\mathbf{Z}^{d}$, let us consider the probability measure $g_{n}:=\sum_{l} g_{n}(l) \delta_{l}$ which puts mass

$$
g_{n}(l):=\frac{\sum_{s} e^{\left\langle m^{\text {inv }}((s-l) / n), l\right\rangle} 1\{(s-l) / n \in A\} P\left\{S_{n}=s\right\}}{\sum_{k} 1\{k / n \in A\} e^{-n I(k / n)}}
$$

at $l \in \mathbf{Z}^{d}$. On $\mathbf{Z}^{d} / \sqrt{n}$, let us define the probability measure $h_{n}:=$ $\sum_{l} g_{n}(l) \delta_{l / \sqrt{n}}$. Notice that

$$
h_{n}(0)=g_{n}(0)=\frac{P\left\{S_{n} \in n A\right\}}{\sum_{k} 1\{k / n \in A\} e^{-n I(k / n)}} .
$$


Lemma 5.4. The characteristic function of $h_{n}$ is given by

$$
\widehat{h}_{n}(\lambda):=\sum_{k} e^{i\langle\lambda, k / \sqrt{n}\rangle} g_{n}(k)=\int e^{-n k(a, \lambda / \sqrt{n})} d \mu_{A, n}(a),
$$

where

$$
k(a, \lambda):=i\langle a, \lambda\rangle+\ln \phi\left(m^{\text {inv }}(a)\right)-\ln \phi\left(m^{\text {inv }}(a)+i \lambda\right) .
$$

P r o o f. Argue as for Lemma 3.4, and use the same change of variable in the summation as in the proof of Lemma 5.3.

Let $\widehat{\psi}_{n}, \widehat{\psi}, \psi_{n}, \psi$ be in the proof of Theorem 2.1. Recall also the Fourier inversion formula (see, e.g., [28]),

$$
h_{n}(0)=(2 \pi)^{-d} \int_{[-\pi \sqrt{n}, \pi \sqrt{n}]^{d}} \widehat{h}_{n}(\lambda) d \lambda
$$

since $h_{n}$ is a lattice distribution with $\operatorname{span} 1 / \sqrt{n}$. Thus

$$
\begin{aligned}
\left|h_{n}(0)-\psi_{n}(0)\right| \leqslant & \int_{[-\pi \sqrt{n}, \pi \sqrt{n}]^{d}}\left|\widehat{h}_{n}(\lambda)-\widehat{\psi}_{n}(\lambda)\right| d \lambda \\
& +\int_{\mathbf{R}^{d} /[-\pi \sqrt{n}, \pi \sqrt{n}]^{d}}\left|\widehat{\psi}_{n}(\lambda)\right| d \lambda \\
= & \int_{[-\pi \sqrt{n}, \pi \sqrt{n}]^{d}}\left|\widehat{h}_{n}(\lambda)-\widehat{\psi}_{n}(\lambda)\right| d \lambda+o(1) \quad \text { as } \quad n \rightarrow \infty
\end{aligned}
$$

As in the continuous case, for $\varepsilon$ small enough,

$$
\int_{[-\varepsilon \sqrt{n}, \varepsilon \sqrt{n}]^{d}}\left|\widehat{h}_{n}(\lambda)-\widehat{\psi}_{n}(\lambda)\right| d \lambda=o(1) \quad \text { as } \quad n \rightarrow \infty .
$$

Let $J_{n}(\varepsilon):=[-\pi \sqrt{n}, \pi \sqrt{n}]^{d} /\left[-\varepsilon \sqrt{n}, \varepsilon \sqrt{n}^{d}\right.$. Then

$$
\int_{J_{n}(\varepsilon)}|\widehat{\psi}(\lambda)| d \lambda=o(1) \quad \text { as } \quad n \rightarrow \infty .
$$

Further, for any $a, \phi\left(m^{\text {inv }}(a)+i \lambda\right) / \phi\left(m^{\text {inv }}(a)\right)$ is the characteristic function of a random variable with lattice distribution $e^{\left\langle m^{\text {inv }}(a), x\right\rangle} d P(x) / \phi\left(m^{\text {inv }}(a)\right)$ on $\mathbf{Z}^{d}$. Thus, $\phi\left(m^{\text {inv }}(a)+i \lambda / \sqrt{n}\right) / \phi\left(m^{\text {inv }}(a)\right)$ is the characteristic function of a lattice distribution concentrated on $\mathbf{Z}^{d} / \sqrt{n}$. Hence,

$$
\sup _{\lambda \in J_{n}(\varepsilon)}\left|\frac{\phi\left(m^{\text {inv }}(a)+i \lambda / \sqrt{n}\right)}{\phi\left(m^{\text {inv }}(a)\right)}\right|=: \eta<1 .
$$

It follows that if $\lambda \in J_{n}(\varepsilon)$, then

$$
\left|\widehat{h}_{n}(\lambda)\right| \leqslant \int\left|\frac{\phi\left(m^{\text {inv }}(a)+i \lambda / \sqrt{n}\right)}{\phi\left(m^{\text {inv }}(a)\right)}\right|^{n} d \mu_{A, n}(a) \leqslant \eta^{n} .
$$

Therefore,

$$
\int_{J_{n}(\varepsilon)}\left|\widehat{h}_{n}(\lambda)\right| d \lambda \leqslant \eta^{n} n^{d / 2} O(1)=o(1) \quad \text { as } \quad n \rightarrow \infty .
$$


Combining (5.1)-(5.4), we infer that

$$
\left|h_{n}(0)-\psi_{n}(0)\right|=o(1) \quad \text { as } \quad n \rightarrow \infty .
$$

Finally, if $\mu_{A, n_{k}}$ converges weakly* to a measure $\mu_{A}$ and $A$ is bounded,

$$
\lim _{n \rightarrow \infty} h_{n_{k}}(0)=\psi(0),
$$

which is the first result we need to prove.

We then prove Corollary 2.5 as we proved Corollary 2.1 but using a subsequence $n_{k}$ such that $c_{n_{k}}$ converges to some $c$. We then conclude the proof as we did for Theorem 2.1.

6. Proof of Theorem 2.3. It follows closely that of Theorem $2.1^{\prime}$ and we shall only sketch it. The tightness of the measures $\mu_{F, n}$ follows as in [30]. The equivalent of Lemma 3.3 is given by

$$
\int \exp \left\langle m^{\text {inv }}\left(\frac{s-x}{n}\right), x\right\rangle \exp \left(n F\left(\frac{s-x}{n}\right)\right) d P\left\{S_{n}=s\right\} d x=\int e^{-n H_{F}(x)} d x .
$$

Thus, we define the density functions

$$
g_{F, n}(x)=\frac{\int \exp \left(\left\langle m^{\text {inv }}((s-x) / n), x\right\rangle+n F((s-x) / n)\right) d P\left\{S_{n}=s\right\}}{\int \exp \left(-n H_{F}(y)\right) d y}
$$

and set $h_{n}(x)=n^{d / 2} g_{F, n}(\sqrt{n} x)$. We then proceed as in the proof of Theorem 2.1.

7. Appendix. To the reader not familiar with differential geometry and who wishes to learn its foundations, we warmly advice to follow the path we took, reading the two illuminating books by Do Carmo, starting with the theory of curves and surface in $\mathbf{R}^{3}$ [24] and moving to the Riemannian geometry [25]. Then, more advanced texts are much easier to read, and we refer for instance to Chavel [21], Gallot, Hulin, and Lafontaine [29] or the treatise by Spivak [42]).

Let $M$ be an $r$-dimensional submanifold of $\mathbf{R}^{d}$ (intuitively, a surface locally homeomorphic to $\mathbf{R}^{r}$ ). Let $N(p), p \in M$, be a smooth vector field, normal to $M$ at $p$, with norm $|N(p)|=1$. Let $X, Y \in T_{p}(M)$ be two tangent vectors at $M$ at $p$. Then there exists a curve $\alpha$ from $(-\varepsilon, \varepsilon)$ to $M$ with $\alpha(0)=p$ and with derivative $\alpha^{\prime}(0)=X$. Since $N(p)=1$, we have $\nabla_{X} N(p):=(N \circ \alpha)^{\prime}(0)$ is orthogonal to $N(p)$ (if $r=d-1$, this forces to have $\left.\nabla_{X} N(p) \in T_{p}(M)\right)$. Let $\left(\nabla_{X} N(p)\right)^{\top}$ be the projection of $\nabla_{X} N(p)$ onto $T_{p}(M)$. The map $\mathscr{W}_{p}: X \in T_{p}(M) \mapsto-\left(\nabla_{X} N(p)\right)^{\top} \in T_{p}(M)$ is the Weingarten map of the immersion $M \in \mathbf{R}^{d}$ relative to the direction $N(p)$ (it can be seen that it depends only on $N(p)$ and not on its extension over a neighborhood of $p$ ). It induces a symmetric bilinear form 
$(X, Y) \in T_{p} M \mapsto\left\langle\mathscr{W}_{p} X, Y\right\rangle$ called the second fundamental form of the immersion $M \subset \mathbf{R}^{d}$. The Gauss-Kronecker curvature at $p$ is just the determinant of the Weingarten map at $p$. One can prove that it corresponds to the following. For each direction $\tau$ in $T_{p}(M)$, let $r(\tau)$ be the radius of the circle in the plane given by $N(p)$ and $\tau$, which is tangent to $M$ at $p$. Let $\tau_{1}$ be the direction in which $r(\tau)$ is minimal (if there is no unique minimum, pick any minimum). If $\tau_{i}$ is defined, define $\tau_{i+1}$ to be the direction orthogonal to $\tau_{1}, \ldots, \tau_{i}$ such that $r(\tau)$ is minimal. Then $\operatorname{det} \mathscr{W}_{p}=\prod_{1 \leqslant i \leqslant r} 1 / r\left(\tau_{i}\right)$. Thus we see that the Gauss-Kronecker curvature measures indeed some kind of curvature of the manifold. The $1 / r\left(\tau_{i}\right)$ 's turn also to be the eigenvalues of $\mathscr{W}_{p}$ associated with the eigenvectors $\tau_{i}$. The eigenvectors are called the principal directions of the manifold.

Let us now consider a manifold $M:=\left\{(v, g(v)): v \in U \subset \mathbf{R}^{r}\right\}$ in $\mathbf{R}^{r+1}$, where $g$ is a real-valued function with $g(0)=0$ and $D g(0)=0$. Then writing $W_{v}:=\operatorname{Hess} g(v)$, we have $g(v)=\frac{1}{2}\left\langle W_{0} v, v\right\rangle+O\left(|v|^{3}\right)$ for small $v$. Denote by $\omega^{1}, \ldots, \omega^{r+1}$ the canonical basis of $\mathbf{R}^{r+1}$. If $p(s)=(v(s), g(v(s)))$ is a curve on $M$, then $p^{\prime}(s)=\left(v^{\prime}(s), D g_{v(s)} v^{\prime}(s)\right) \in T_{p(s)}(M)$. Thus, viewing $D g_{v(s)}$ as a vector, we infer that $\left(-D g_{v(s)}, 1\right)$ is normal to $M$ at $p(s)$, and in particular $\omega^{r}$ is normal at 0 . It follows that

$$
\begin{aligned}
\mathscr{W}_{0}\left(v^{\prime}(0)\right) & =-\left.\frac{d}{d s}\left(-D g_{v(s)}, 1\right)\right|_{s=0}=-\left.\left(-D^{2} g_{v(s)} v^{\prime}(s), 0\right)\right|_{s=0} \\
& =\left(W_{0} v^{\prime}(0), 0\right) .
\end{aligned}
$$

Hence the Weigarten map at $0, \mathscr{W}_{0}$, is just the Hessian of $g$ at 0 .

We now do a similar calculation for the manifold $\Lambda_{c}$. We believe this should be somewhere in the literature on large deviations or on exponential families, but we have not been able to find it. A result in the same spirit is in Remark 3 following Proposition 4.1 in [33], where it is shown that the inverse of the variance function is the second fundamental form of the surface $(x, I(x))$.

First, let $t(s)$ be such that $m(t(s))$ is a curve on $\Lambda_{c}$. Then $I(m(t(s)))=$ $c=\langle m(t(s)), t(s)\rangle-\ln \phi(t(s))$ and

$$
\frac{d}{d s} I(m(t(s)))=0=\left\langle\frac{d}{d s} m(t(s)), t(s)\right\rangle .
$$

But since $m(t(s)) \in \Lambda_{c}, \frac{d}{d s} m(t(s)) \in T_{m(t(s))} \Lambda_{c}$. Since any vector in the tangent space $T_{p}\left(\Lambda_{c}\right)$ is of the form $\left.\frac{d}{d s} m(t(s))\right|_{s=0}$ for a curve $t(s) \in \Lambda_{c}$ with $m(t(0))=p$, (7.1) shows that $t$ is normal to $\Lambda_{c}$ at $m(t)$. In other words, if $p \in \Lambda_{c}$, then $m^{\text {inv }}(p)$ is normal to $\Lambda_{c}$ at $p$.

Then $N(s)=t(s) /|t(s)|$ is a normal field on $\Lambda_{c}$, pointing outward $\Gamma_{c}$. We have

$$
\frac{d}{d s}\langle t(s), t(s)\rangle^{-1 / 2}=\frac{-\left\langle t^{\prime}, t\right\rangle}{\langle t, t\rangle^{3 / 2}}(s)=-\frac{1}{|t|}\left\langle\frac{t^{\prime}}{|t|}, \frac{t}{|t|}\right\rangle(s) .
$$


Thus

$$
\frac{d}{d s} N(s)=\frac{t^{\prime}}{|t|}-\frac{t}{|t|}\left\langle\frac{t^{\prime}}{|t|}, \frac{t}{|t|}\right\rangle=\left(\frac{t^{\prime}}{|t|}\right)^{\top}
$$

since $t$ is normal, and where ${ }^{\top}$ means that we project the vector from $\mathbf{R}^{d}$ to the tangent space $T_{m(t(s))} \Lambda_{c}$. But since $\frac{d}{d s} m(t(s))=H(t(s)) t^{\prime}(s),(7.2)$ can be rewritten as

$$
\nabla_{H(t(s)) t^{\prime}(s)} N(s)=\left(\frac{t^{\prime}}{|t|}\right)^{\top}
$$

i.e., if $\mathscr{W}_{p}$ is the Weingarten map at $p \in \Lambda_{c}$ associated with the outward normal $m^{\text {inv }}(p) /\left|m^{\text {inv }}(p)\right|$, we have (setting $u=t^{\prime}$ )

$$
\mathscr{W}_{m(t)}(H(t) u)=-\left(\frac{u}{|t|}\right)^{\top}=-\frac{u^{\top}}{|t|} .
$$

Since any $v \in T_{p} \Lambda_{c}$ can be written as $H(t(s)) t^{\prime}(s)$ for some curve $t(s)$, we obtain that

$$
\mathscr{W}_{p}(v)=-\frac{\left(H\left(m^{\mathrm{inv}}(p)\right)^{-1} v\right)^{\top}}{\left|m^{\mathrm{inv}}(p)\right|}=-\frac{\left(V(p)^{-1} v\right)^{\top}}{\left|m^{-1}(p)\right|} .
$$

Now, notice that if $w \in T_{p} \Lambda_{c}$, then

$$
\left\langle\mathscr{W}_{p}(v), w\right\rangle=\left\langle\frac{V(p)^{-1} v}{\left|m^{\text {inv }}(p)\right|}, w\right\rangle .
$$

Thus the second fundamental form is the quadratic form (in $T_{p}\left(\Lambda_{c}\right)$ ) associated to the matrix $\left|m^{\text {inv }}(p)\right|^{-1} V(p)^{-1}$. However, for calculating the Gauss-Kronecker curvature of $\Lambda_{c}$, be careful that in general $\operatorname{det}\left(\left|m^{\text {inv }}(p)\right|^{-1} V(p)^{-1}\right) \neq \operatorname{det} \mathscr{W}_{p}$, the bilinear form $|m(p)|^{-1} V(p)^{-1}$ is acting on $\mathbf{R}^{d}$ while $\mathscr{W}_{p}$ is defined on $T_{p} \Lambda_{c} \equiv \mathbf{R}^{d-1}$.

We now consider two submanifolds $M_{1}, M_{2}$ of dimension $r-1$ in $\mathbf{R}^{r}$, tangent at a point $p$, i.e., such that $T_{p} M_{1}=T_{p} M_{2} \equiv \mathbf{R}^{r-1}$. Let $\omega^{1}, \ldots, \omega^{r-1}$ be an orthonormal basis in $T_{p} M_{i}$, and $\omega^{r}$ be a unit normal vector to $M_{1}$ and $M_{2}$ at $p$. We identify $\mathbf{R}^{r}$ with $T_{p} M_{i} \oplus \omega^{r} \mathbf{R}$. Locally, in the basis $\left(\omega^{1}, \ldots, \omega^{r}\right)$ we can identify $M_{i}$ with

$$
\left\{p+\left(x, f_{i}(x)\right):|x| \leqslant \varepsilon\right\},
$$

where $x=\sum_{1 \leqslant i \leqslant r-1} x_{i} \omega^{i},(x, u)=x+u \omega^{r}$, and $D f_{i}(0)=0$ (i.e., the manifold looks locally like a paraboloid). We then view $M_{2}$ from $M_{1}$. The vertical at $p(x):=\left(x, f_{1}(x)\right) \in M_{1}$ is given by the normal vector $\nu(x):=\left(-D f_{1}(x), 1\right)$. Thus, from $M_{2}$, points in $M_{1}$ are of the form

$$
p(x)+\alpha(x) \nu(x)=\left(x-\alpha(x) D f_{1}(x), f_{1}(x)+\alpha(x)\right) .
$$

Thus, we have

$$
f_{2}\left(x-\alpha(x) D f_{1}(x)\right)=f_{1}(x)+\alpha(x) .
$$


Expanding this equality in a neighborhood of 0 yields

$$
D \alpha(0)=0 \quad \text { and } \quad D^{2} \alpha(0)=D^{2} f_{2}(0)-D^{2} f_{1}(0) .
$$

Hence, viewed from $M_{2}$, the manifold $M_{1}$ has Gauss-Kronecker curvature $\left|D^{2} \alpha(0)\right|=\left|D^{2} f_{2}(0)-D^{2} f_{1}(0)\right|$.

\section{REFERENCES}

1. Andriani C., Baldi P. Sharp estimates of deviations of the sample mean in many dimensions. - Ann. Inst. H. Poincaré Probab. Statist., 1997, v. 33, № 3, p. 371-385.

2. Azencott $R$. Grandes déviations et applications. - Lecture Notes in Math., 1980, v. 774 , p. 1-176.

3. Bahadur R. R., Ranga Rao R. On deviations of the sample mean. - Ann. Math. Statist., 1960, v. 31, p. 1015-1027.

4. Bahadur R. R., Zabell S. L. Large deviations of the sample mean in general vector spaces. - Ann. Probab., 1979, v. 7, № 4, p. 587-621.

5. Balkema A. A., Geluk J. L., de Haan L. An extension of Karamata's Tauberian theorem and its connection with complementary convex functions. - Quart. J. Math. Oxford, 1979, v. 30, № 120, p. 385-416.

6. Barndorff-Nielsen O. Information and Exponential Families in Statistical Theory. Chichester: Wiley, 1978, 238 p.

7. Bingham N. H., Goldie C. M., Teugels J. L. Regular Variation. Cambridge: Cambridge University Press, 1989, 494 p.

8. Bleistein N., Handelsman R. A. Asymptotic Expansions of Integrals. New York: Holt, Rinehart and Winston, 1975, $425 \mathrm{p}$.

9. Bolthausen E. Laplace approximations for sums of independent random vectors. Probab. Theory Related Fields, 1986, v. 72, № 2, p. 305-318.

10. Bolthausen E. Laplace approximations for sums of independent random vectors. II. Degenerate maxima and manifold of maxima. - Probab. Theory Related Fields, 1987, v. 76 , № 2 , p. 167-206.

11. Borovkov A. A., Mogulskii A. A. Large deviations and testing statistical hypothesis. I. Large deviations of sums of random vectors. - Sib. Adv. Math., 1992, v. 2, № 3, p. $53-120$.

12. Borovkov A. A., Mogulskii A. A. Large deviations and testing satistical hypothesis. II. Large deviations of the maximum points of random fields. - Sib. Adv. Math., 1992, v. 2 , № 4 , p. $43-72$.

13. Borovkov A. A., Mogulskii A. A. Large deviations and testing satistical hypothesis. III. The statistical invariance principle and the laws of conservation. - Sib. Adv. Math., 1993, v. 3, № 2, p. 14-80.

14. Боровков А.А., Могульский А. А. Интегро-локальные предельные теоремы для сумм случайных векторов, включаюшие большие уклонения. I, II. - Теория вероятн. и ее примен., 1998, т. 43 , в. 1, с. 3-17; 2000 , т. 45, в. 1, с. 5-29.

15. Боровков A. A., Рогозин Б. А. О центральной предельной теореме в многомерном случае. - Теория вероятн. и ее примен., 1965, т. 10, в. 1, с. 61-69.

16. Breitung K., Hohenbichler M. Asymptotic approximations for multivariate integrals with an application to multinormal probabilities. - J. Multivariate Anal., 1989, v. 30, № 1, p. 80-97.

17. Brown L. D. Fundamentals of Statistical Exponential Families with Applications in Statistical Decision Theory. Hayward, CA: Institute of Mathematical Statistics, 1986, $283 \mathrm{p}$.

18. De Bruijn N. G. Pairs of slowly oscillating functions occuring in asymptotic problems concerning the Laplace transform. - Nieuw Arch. Wisk., 1959, v. 7, p. 20-26.

19. Bucklew J. A. Large Deviation Techniques in Decision, Simulation, and Estimation New York: Wiley, 1990, 270 p. 
20. Chaganty N.R., Sethuraman J. Multidimensional large deviation local limit theorems. - J. Multivariate Anal., 1986, v. 20, № 2, p. 190-204.

21. Chavel I. Riemannian Geometry: A Modern Introduction. Cambridge: Cambridge Univ. Press, 1993, 386 p.

22. Dembo A., Zeitouni O. Large Deviations Techniques and Applications. Boston, MA: Jones and Bartlett, 1993, $346 \mathrm{p}$.

23. Deuschel J.-D., Stroock D. Large Deviations. Boston, MA: Academic Press, 1989, $307 \mathrm{p}$.

24. do Carmo M. P. Differential Geometry of Curves and Surfaces. Englewood Cliffs, NJ: Prentice Hall, 1976, 503 p.

25. do Carmo M. P. Riemannian Geometry. Boston, MA: Bikhäuser, 1992, 300 p.

26. Donsker M., Varadhan S.R.S. Asymptotic evaluation of certain Markov process expectations for large time. III. - Comm. Pure Appl. Math., 1976, v. 29, № 4, p. 389461.

27. Ellis R. S. Entropy, Large Deviations, and Statistical Mechanics. New York: SpringerVerlag, 1985, $364 \mathrm{p}$.

28. Феллер $W$. Введение в теорию вероятностей и ее приложения. Т. 2. М.: Мир, $1984,752 \mathrm{c}$.

29. Gallot S., Hulin D., Lafontaine J. Riemannian Geometry. Berlin: Springer-Verlag, 1993.

30. Hwang C.-R. Laplace's method revisited: weak convergence of probability measures. - Ann. Probab., 1980, v. 8, №6, p. 1177-1182.

31. Iltis $M$. Sharp asymptotics of large deviations in $\mathbf{R}^{d}$. - J. Theoret. Probab., 1995, v. 8 , № 3, p. 501-522.

32. Jensen J. L. Saddlepoint Approximations. New York: Clarendon Press, Oxford Univ. Press, 1995, $332 \mathrm{p}$.

33. Letac G. Lectures on Natural Exponential Families and their Variance Functions. Rio de Janeiro: Instituto de Matemática Pura e Aplicada (IMPA), 1992, 128 p.

34. Nagaev A. V. Lower bounds for large deviations in $\mathbf{R}^{d}, d>1$. - Math. Nachr., 1991, v. 154 , p. $41-49$.

35. Ney $P$. Dominating points and the asymptotics of large deviations for random walk on $\mathbf{R}^{d}$. - Ann. Probab., 1983, v. 11, № 1, p. 158-167.

36. Петров $B . B$. О вероятностях больших уклонений сумм независимых случайных величин. - Теория вероятн. и ее примен., 1965 , т. 10, в. 2, с. 310-322.

37. Петров В.В., Широкова И. В. Экспоненциальная скорость сходимости в законе больших чисел. - Вестник Ленинградского ун-та, сер. матем., мех., астроном., 1973, № 2, c. 155-157.

38. Рихтер B. Локальные предельные теоремы для больших уклонений. - Теория вероятн. и ее примен., 1957, т. 2, в. 2, с. 214-229.

39. Рихтер B. Многомерные локальные предельные теоремы для больших уклонений. - Теория вероятн. и ее примен., 1958, т. 3, в. 1, с. 107-114.

40. Ruben $H$. An asymptotic expansion for the multivariate normal distribution and Mills' ratio. - J. Res. Nat. Bur. Standards Sect. B, 1964, v. 68B, p. 3-11.

41. Slaby M. On the upper bound for large deviations of sums of i.i.d. random vectors. Ann. Probab., 1988, v. 16, № 3, p. 978-990.

42. Spivak M. A Comprehensive Introduction to Differential Geometry. V. 1-5. Boston: Publish or Perish, 1979, 668 p., 423 p., 466 p., 561 p., 661 p.

43. Stroock $D$. An Introduction to the Theory of Large Deviations. New York: SpringerVerlag, 1984, $196 \mathrm{p}$.

44. Vinogradov V. Refined Large Deviation Limit Theorems. Harlow: Longman, 1995, $212 \mathrm{p}$.

45. Wagner E. Ein reeller Tauberscher Satz für die Laplace-Transformation. - Math. Nachr., 1968, v. 36, p. 323-331.

46. Weyl H. On the volume of tubes. - Amer. J. Math., 1939, v. 61, p. 461-472. 